Provided for non-commercial research and education use. Not for reproduction, distribution or commercial use.

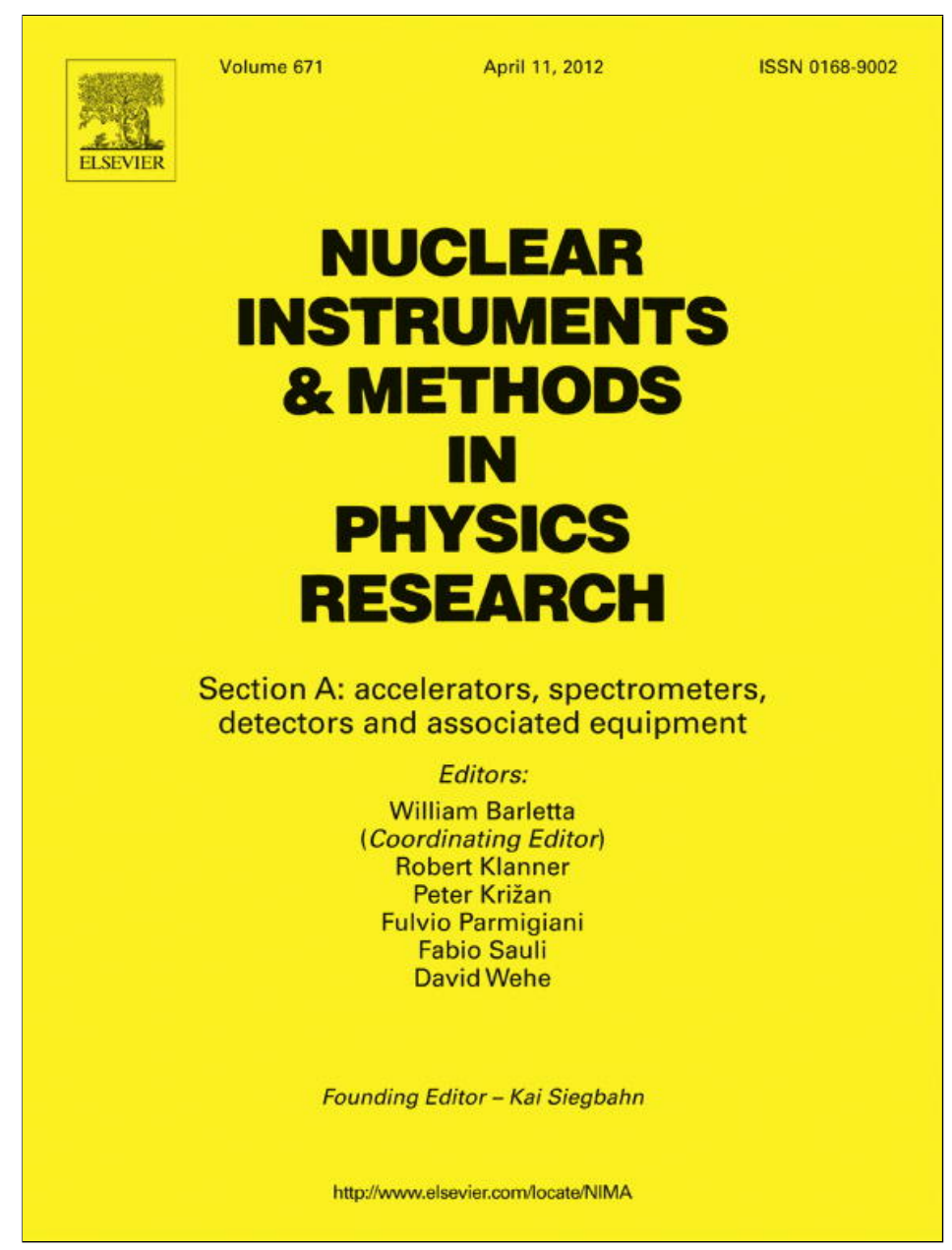

This article appeared in a journal published by Elsevier. The attached copy is furnished to the author for internal non-commercial research and education use, including for instruction at the authors institution and sharing with colleagues.

Other uses, including reproduction and distribution, or selling or licensing copies, or posting to personal, institutional or third party websites are prohibited.

In most cases authors are permitted to post their version of the article (e.g. in Word or Tex form) to their personal website or institutional repository. Authors requiring further information regarding Elsevier's archiving and manuscript policies are encouraged to visit:

http://www.elsevier.com/copyright 


\title{
Optimizations of transverse projected emittance at the photo-injector test facility at DESY, location Zeuthen
}

\author{
S. Rimjaem ${ }^{\text {a,* }}$, F. Stephan ${ }^{\text {a }}$, M. Krasilnikov ${ }^{\text {a }}$, W. Ackermann ${ }^{\text {b }}$, G. Asova ${ }^{\text {a,1 }}$, J. Bähr $^{\text {a }}$, E. Gjonaj ${ }^{\text {b }}$, \\ H.J. Grabosch ${ }^{a}$, L. Hakobyan ${ }^{a}$, M. Hänel ${ }^{a, 2}$, Y. Ivanisenko ${ }^{a, 3}$, M. Khojoyan $^{a, 4}$, G. Klemz ${ }^{a}$, S. Lederer ${ }^{c}$, \\ M. Mahgoub ${ }^{a}$, P. Michelato ${ }^{d}$, L. Monaco ${ }^{d}$, M. Nozdrin ${ }^{\mathrm{a}, 5}$, B. O’Shea $^{\mathrm{a}, 6}$, M. Otevrel ${ }^{\mathrm{a}}$, B. Petrosyan ${ }^{\mathrm{a}}$, \\ D. Richter ${ }^{\mathrm{e}}$, J. Rönsch-Schulenburg ${ }^{\mathrm{f}}$, D. Sertore ${ }^{\mathrm{d}}$, S. Schreiber ${ }^{\mathrm{c}}$, S. Schnepp ${ }^{\mathrm{b}}$, A. Shapovalov ${ }^{\mathrm{a}, 7}$, \\ R. Spesyvtsev ${ }^{\mathrm{a}, 8}$, L. Staykov ${ }^{\mathrm{a}, 9}$, G. Vashchenko $^{\mathrm{a}}$, T. Weiland ${ }^{\mathrm{b}}$, I. Will ${ }^{\mathrm{g}}$
}

a Deutsches Elektronen-Synchrotron (DESY), Platanenallee 6, 15738 Zeuthen, Germany

b Technische Universtät Darmstadt, Schoßgartenstraße 8, 64289 Darmstadt, Germany

${ }^{c}$ Deutsches Elektronen-Synchrotron (DESY), Notkestraße 85, 22607 Hamburg, Germany

${ }^{\mathrm{d}}$ Istituto Nazionale di Fisica Nucleare, Sezione di Milano - LASA, Via F.lli Cervi 201, 20090 Segrate Milano, Italy

e Helmholtz-Zentrum Berlin, Hahn-Meitner-Platz 1, 14109 Berlin, Germany

${ }^{f}$ Universität Hamburg, Luruper Chaussee 149, 22761 Hamburg, Germany

${ }^{g}$ Max-Born-Institut, Max-Born-Straße 2A, 12487 Berlin, Germany

\section{A R T I C L E I N F O}

\section{Article history:}

Received 9 November 2011

Received in revised form

7 December 2011

Accepted 26 December 2011

Available online 30 December 2011

Keywords:

Photo-injector

RF-gun

Transverse projected emittance

Free-electron laser injector

\begin{abstract}
A B S T R A C T
High brightness electron sources for linac based short-wavelength free-electron lasers are developed and optimized for small transverse projected emittance at the photo-injector test facility at DESY, location Zeuthen (PITZ). A major part of the measurement program at PITZ is dedicated to transverse phase space optimization in order to fulfill the requirements of the European X-ray free-electron laser (European XFEL). A laser-driven RF-gun, treated with a dry-ice sublimation-impulse cleaning technique, a new photocathode laser system allowing short rise and fall times of the flat-top temporal distribution as well as several new diagnostic components have been installed at PITZ in 2008. The electrons generated via the photo-effect at a cesium telluride $\left(\mathrm{Cs}_{2} \mathrm{Te}\right)$ cathode are accelerated by a 1.6 cell L-band RF-gun cavity with a maximum accelerating gradient at the cathode of about $60 \mathrm{MV} / \mathrm{m}$. The transverse projected emittance is measured using a single slit scan technique. In the 2008-2009 run period, a detailed characterization of the projected transverse emittance was performed at different operating conditions. Optimizations and measurement results as well as simulation predictions of the transverse projected emittance for bunch charges of $1,0.5$, 0.25 and $0.1 \mathrm{nC}$ are presented and discussed in this paper. The geometric mean of the normalized projected rms emittance in both transverse directions for an electron bunch charge of $1 \mathrm{nC}$ was measured to be $0.89 \pm 0.01 \mathrm{~mm}$ mrad for a $100 \% \mathrm{rms}$ phase-space distribution.
\end{abstract}

(c) 2011 Elsevier B.V. All rights reserved.

\section{Introduction}

Linac based free-electron laser (FEL) light sources like the freeelectron laser at DESY (FLASH) [1], the European X-ray free-electron

\footnotetext{
* Corresponding author. Tel.: +4933 7627 7185; fax: +49337627 7330 E-mail addresses: r.sakhorn@gmail.com,

sakhorn.rimjaem@desy.de (S. Rimjaem).

${ }^{1}$ On leave from INRNE, Sofia, Bulgaria.

2 Currently at Siemens AG, Rudolstadt, Germany.

${ }^{3}$ On leave from IERT, NAS, Kharkiv, Ukraine.

${ }^{4}$ On leave from YERPHI, Yerevan, Armenia.

${ }^{5}$ On leave from JINR, Dubna, Russia.

${ }^{6}$ On leave from UCLA, Los Angeles, California, USA.

7 On leave from NRNU MEPhi, Moscow, Russia.

${ }^{8}$ Currently at University College London, UK.

${ }^{9}$ Currently at Advanced Energy Systems, Inc., Princeton, NJ, USA.
}

laser (European XFEL) [2], the linac coherent light source (LCLS) at SLAC [3], the Spring-8 Compact SASE source (SCSS) X-FEL [4], the free-electron laser for multidisciplinary investigations (FERMI) at Elettra [5], and the Swiss free-electron laser (SwissFEL) [6] require electron beams of high quality for both, the transverse and longitudinal phase spaces. Research activities at the photo-injector test facility at DESY, location Zeuthen (PITZ) combine development, characterization, and optimization of photo-injector electron sources to produce electron beams with parameters yielding the requirements for FLASH and the European XFEL. Wavelength and gain of the self amplified spontaneous emission (SASE) FEL process are defined by peak current, energy spread, and transverse emittance of the electron beam for a certain undulator period and length. As an example, a nice overview on high gain FEL can be found in Ref. [7]. The peak current and energy spread are properties which can be improved during the 


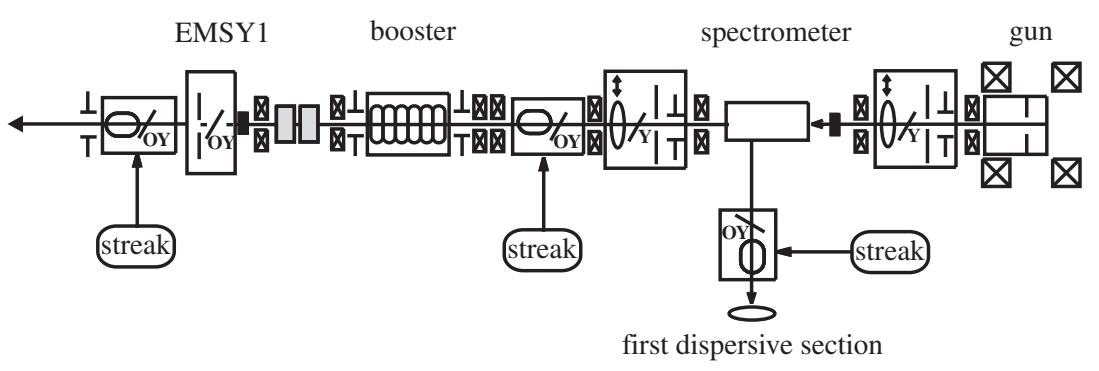

beam dump spectrometer EMSY3 spectrometer EMSY2

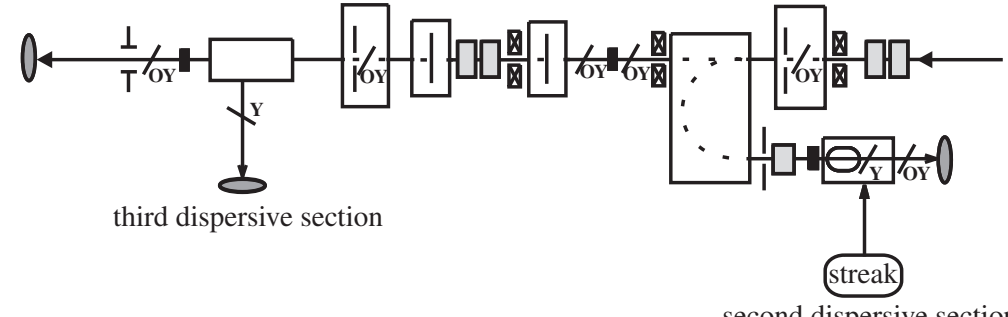

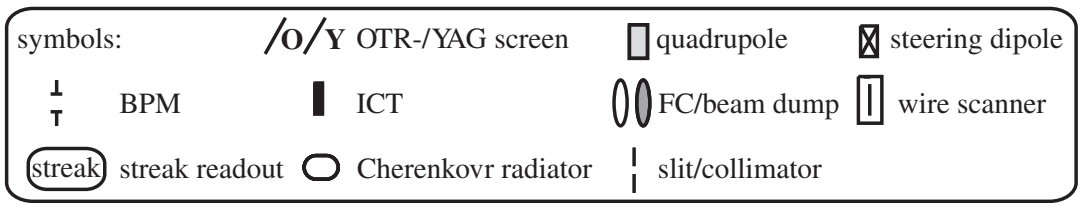

Fig. 1. Schematic diagram of the PITZ diagnostics beam line in 2008-2009 including electron gun, booster cavity, dispersive sections, optical transition radiation and YAG screens $(\mathrm{O} / \mathrm{Y})$, and emittance measurement system (EMSY) stations.

beam transport before the electron beam enters the undulator, e.g. by using accelerating structures or bunch compressors. On the contrary, the transverse emittance of the beam has to be optimized at the source since it cannot be improved anymore after the beam exits the injector which includes the gun and the next accelerating module except an emittance exchange system is installed downstream. The emittance exchange system can improve the transverse emittance but on the expense of the longitudinal emittance [8-10].

The required transverse slice emittance of an electron beam with a bunch charge of $1 \mathrm{nC}$ at the undulator entrance of the European XFEL is $1.4 \mathrm{~mm}$ mrad. With some room for the degradation of the slice emittance from the gun to the undulator and some safety margin, a normalized projected transverse emittance of $0.9 \mathrm{~mm}$ mrad at the photo-injector exit is required [2]. Since the production of electron beams with such a small emittance is the most challenging research task at PITZ, a major part of the measurement program is devoted to the transverse emittance optimization. Experimental results of the projected transverse emittance from the setup in 2007 yielded a measured geometric mean emittance ${ }^{10}$ of $1.26 \pm 0.13 \mathrm{~mm}$ mrad for a $100 \% \mathrm{rms}$ phase space distribution [11]. There are several improvements and changes of the PITZ beam transport line compared to the setup in 2007 with the goal to improve the electron source properties and to extend the capability of the facility for beam characterization. Since this upgrade was significant, a new machine optimization became necessary. Measurement results with the improved setup, described in this paper, show the possibility to achieve and surpass the required projected transverse emittance for the European XFEL photo-injector.

\footnotetext{
${ }^{10}$ The geometric mean emittance is given as the geometric mean of the normalized transverse projected rms emittance averaged over the two transverse planes.
}

\section{PITZ setup and machine preparations}

The PITZ setup in 2008-2009 (Fig. 1) consisted of a laser driven RF-gun, a normal conducting TESLA type booster cavity and various beam diagnostic systems. The main changes compared to the setup in 2007 included a new gun cavity, the installation of a new photocathode laser system, a position shift of the booster cavity, the installation of a new $180^{\circ}$ dipole spectrometer with a corresponding dispersive section, the installation of quadrupole magnets, a position shift of emittance measurement systems corresponding to the shift of the booster cavity, and the modification of several diagnostic components i.e. improvement of TV and video systems, re-design of a diagnostic cross and a spectrometer dipole in the low energy section. More details of the upgrades in the PITZ beam line can be found in Refs. $[12,13]$.

\subsection{Electron gun}

The electron gun in the PITZ setup is a 1.6 cell L-band normal conducting RF cavity with a $\mathrm{Cs}_{2} \mathrm{Te}$ photocathode located at the backplane of the cavity. The photocathodes are produced by INFN-LASA and have a high quantum efficiency and a long life time [14-16]. The RF-gun is powered by a $10 \mathrm{MW}$ klystron via a coaxial RF coupler and has a resonance frequency of $1.3 \mathrm{GHz}$. The RF pulse length is up to $900 \mu$ s with a repetition rate of $10 \mathrm{~Hz}$. The duty cycle is almost $1 \%$. The gun is designed for an average power of up to $50 \mathrm{~kW}$. It is surrounded by a main and a bucking solenoid magnet. The main solenoid is used to focus the beam to counteract the repulsion due to the space-charge force and to focus the beam at the entrance of the booster cavity, while the bucking solenoid is used to cancel the residual field of the main solenoid at the photocathode plane (see Ref. [11] and references therein). The new gun cavity (gun prototype 4.2), which was used in the setup described here, was designed with similar geometry to the previous gun [11] but with an improvement in the cooling system for operation with high RF peak power and long pulse trains. Low 
level RF measurements and tuning of the gun cavity were performed after the fabrication was completed. The gun was mechanically tuned by slightly deforming the cavity end walls to reach the desired resonance frequency of $1.3 \mathrm{GHz}$ at an operating temperature of $55{ }^{\circ} \mathrm{C}$. The peak accelerating field at the cathode was tuned to be about $5 \%$ higher than the field at the center of the second cell [17].

The gun cavity's interior surface was prepared using a dry-ice sublimation-impulse cleaning technique [18]. It includes several thermo-mechanical and chemical processes. This cleaning technique is able to clean a cavity surface by removing particles with a size down to $100 \mathrm{~nm}$. By applying this cleaning technique to the RF-gun, a significant dark current reduction by a factor of 10 was achieved compared to the previous guns where high-pressure water rinsing was used $[12,19]$. The reduced amount of dark current improves the cathode lifetime for operation with high electric peak fields and allows lower vacuum pressures to be maintained during RF operation. After successful commissioning we demonstrated the required RF parameters for the injector of the European XFEL: an average RF power of $50 \mathrm{~kW}$ with an RF power of $7.1 \mathrm{MW}$, an RF pulse length of $700 \mu$ s and a repetition rate of $10 \mathrm{~Hz}$ [19].

\subsection{Photocathode laser system}

A new photocathode laser system was developed by the MaxBorn Institute (MBI) and was installed at PITZ in 2008. The laser system is based on a diode pumped $\mathrm{Yb}$ :KGW laser oscillator, an Yb:YAG regenerative amplifier, and a two-stage Yb:YAG double pass amplifier [20]. It can produce laser pulse trains of up to 800 micro-pulses with $1 \mathrm{MHz}$ frequency at $10 \mathrm{~Hz}$ repetition rate, which leads to a spacing of $1 \mu$ s between the pulses. The UV output pulses have a wavelength of $257 \mathrm{~nm}$ and an energy of $\sim 10 \mu \mathrm{J}$ per micro pulse. Due to the high quantum efficiency (QE) of the $\mathrm{Cs}_{2} \mathrm{Te}$ cathode of $5-10 \%$, not more than $100 \mathrm{~nJ}$ of laser energy is sufficient to produce high charge electron beams of $1 \mathrm{nC}$. The large overhead is required for transverse shaping and as a reserve in case of $\mathrm{QE}$ degradation. The charge of the emitted electron bunches can be changed by adjusting the laser energy with a remote controlled variable attenuator. The number of micro-pulses in a pulse train can be changed remotely from 1 to 800 .

The previous laser system had a so-called "M-shape" temporal profile of maximum 24 ps FWHM pulse length with a dip in the middle, and with rise and fall times of $6-8$ ps [22]. Beam dynamics simulations suggest that steepening the edges of the laser pulse decreases the contribution from the large slice emittances in the head and tail of the electron bunch emitted from the photocathode and therefore limits the growth of the projected emittance due to the space-charge force [21]. Simulation results reveal that a decrease of the rise and fall time from $6 \mathrm{ps}$ to $2 \mathrm{ps}$ leads to a reduction of the transverse projected emittance of about 30\% [22].

The new laser system was thus designed to allow faster rise and fall times of the longitudinal flat-top shape. A pulse shaper based on multicrystal birefringent filters in the new laser system can transform the initial Gaussian pulse shape into a temporal flat-top shape with rise and fall times as short as $2 \mathrm{ps}$ and a maximum pulse length of 25 ps FWHM [20]. The length of the temporal profile can be adjusted in order to study the dependence of the electron beam properties on the laser pulse length. The temporal profile of the UV output pulses is measured with an optical sampling system using an optical cross-correlation technique. A single UV pulse train ( $>400$ micro-pulses) is sampled with the IR sub-ps laser pulses of the oscillator. This allows a measurement of the longitudinal shape with a rate of $10 \mathrm{~Hz}$. The

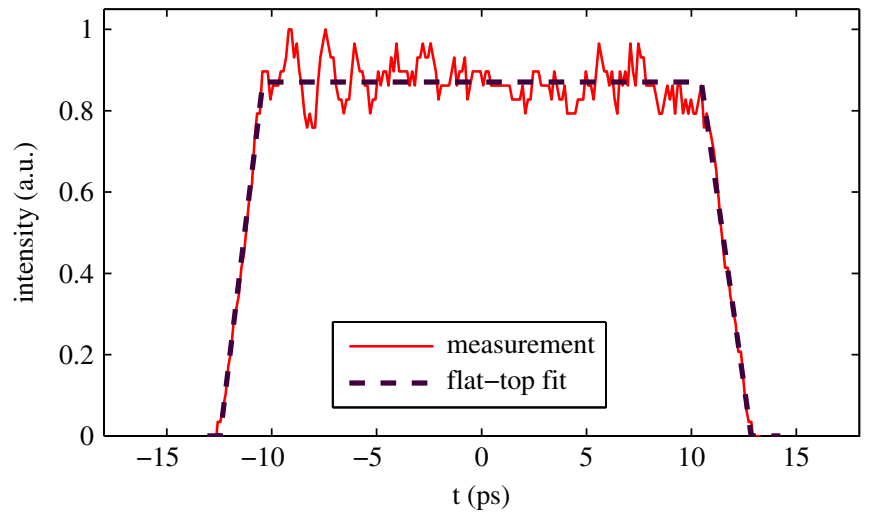

Fig. 2. Example of a photocathode laser temporal profile measured with the optical sampling system (red solid line) in comparison with the flat-top fit (black dash line). The FWHM pulse width is about 23 ps with rise and fall-time of 2 ps each. (For interpretation of the references to color in this figure legend, the reader is referred to the web version of this article.)

obtained shape is in good agreement with those obtained with a single shot measurement of one micro pulse using a high resolution streak camera [23]. The resolution of the measured temporal shape of the laser pulses is better than 1 ps [20]. Laser temporal profiles used in the measurements mentioned in this paper were flat-top pulses with 20-25 ps FWHM and 2-4 ps rise and fall time. Fig. 2 shows an example of a measured temporal laser profile (blue) in comparison with the flat-top fit (red) for a pulse length of about 23 ps (FWHM) and rise and fall times of 2 ps.

The transverse laser spot size on the photocathode has an important impact on the transverse emittance. A set of remotely changeable circular beam shaping apertures of different sizes is used to select the central part of a wide Gaussian transverse profile of the laser beam in order to adjust the transverse laser spot size at the cathode. First, the laser beam is imaged onto the beam shaping aperture and then the cut beam is imaged onto the cathode. This aims to result in a round and a flat-top transverse shape of the laser beam. The beam shaping apertures have diameter sizes from 0.2 to $3 \mathrm{~mm}$ corresponding to laser rms spot sizes at the cathode from 0.05 to $0.44 \mathrm{~mm}$. The laser transverse profile is monitored using a so-called virtual cathode system. It consists of a beam splitter for reflecting the laser light towards a CCD camera which is located at the cathode-equivalent position. An example of the transverse distribution of the photocathode laser pulse is shown in Fig. 3. The laser intensity profiles across a line cut through the center of the laser spot for both horizontal and vertical plane are shown in Fig. 4. For this example, the laser spot is quite symmetric and the laser intensity is flat at the central part of the transverse distribution. However, some inhomogeneity of the distribution is observed at the boundary of the spot. The homogeneity of the laser transverse distribution for various beam shaping apertures depends on the selected part of the initial Gaussian transverse profile of the laser beam. In emittance optimizations, the effort to achieve the symmetric and homogenous transverse laser distribution has been performed.

\subsection{Tuning of photo-injector}

The emittance measurement quality depends strongly on the machine conditions and the electron beam characteristics. Therefore, a proper setup of the photo-injector is required prior to taking data with a procedure as described in detail in Ref. [11]. The preparation starts with a beam-based alignment of the laser position at the cathode and the main solenoid magnet, with the goal to align the laser spot and the main gun solenoid such that the electron beam is emitted from the cathode on the axis of the 


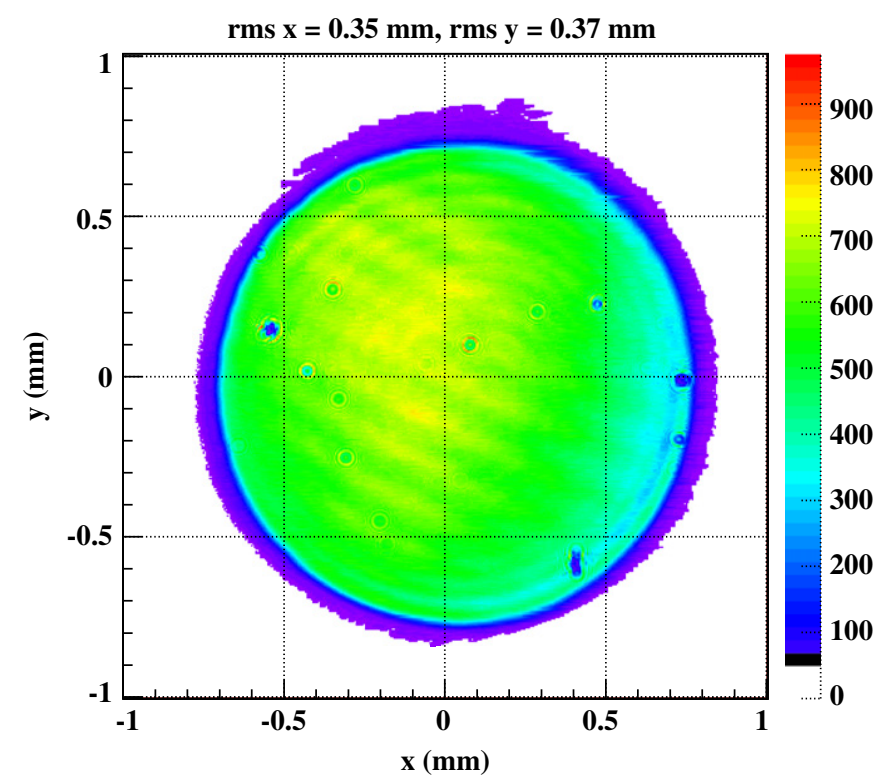

Fig. 3. Example of the transverse distribution of the photocathode laser pulse for a beam shaping aperture resulting an rms spot size of $0.36 \mathrm{~mm}$.

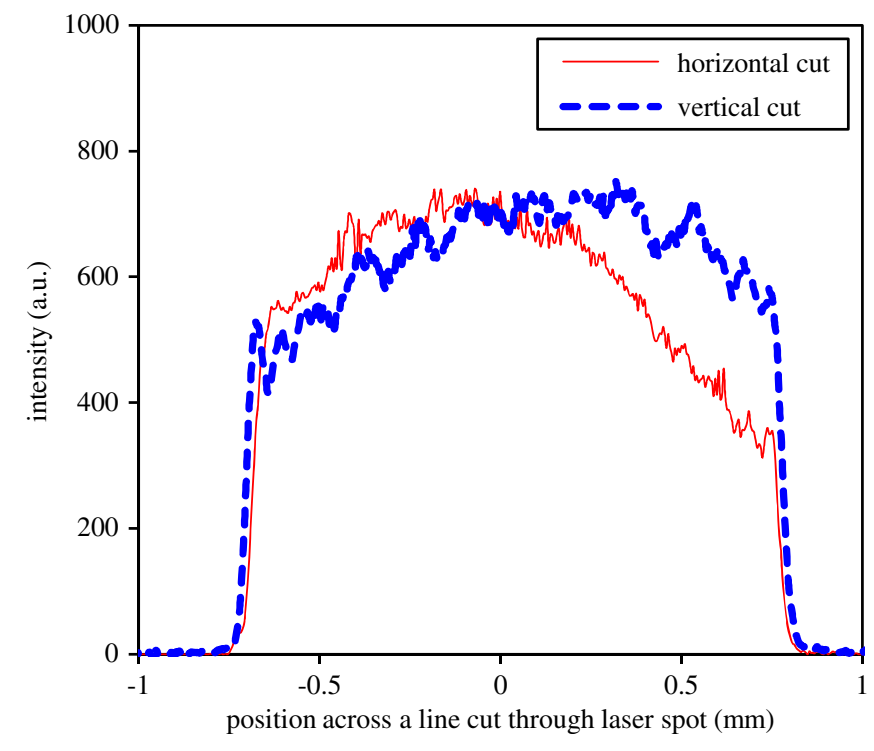

Fig. 4. Centered horizontal and vertical cut profiles of the transverse laser distribution shown in Fig. 3.

electromagnetic field inside the gun cavity, which also has to match with the axis of the solenoid magnetic field. In this case, the electron beam experiences only rotationally symmetric accelerating and focusing fields. Beforehand, checking of the temporal laser profile and the transverse laser size as well as homogeneity of the laser transverse distribution are required. Inhomogeneity of the laser transverse distribution can cause non-symmetric emission of the electron beam. In the next step, the gun RF phase is defined with respect to the laser pulse hitting the cathode. As a reference launch phase the phase with maximum mean momentum gain (MMMG) is chosen. The desired charge per bunch is adjusted by varying the laser intensity at the chosen gun phase. Finally, the booster RF phase is defined also with respect to the maximum mean momentum gain condition.

Electron bunches are emitted when the laser pulses hit the cathode. Then, they are accelerated by the electromagnetic field inside the RF-gun cavity and exit the gun with a beam momentum depending on the chosen emission phase and the gradient of the accelerating field. The beam momentum and the momentum spread are measured at about $1 \mathrm{~m}$ downstream of the gun cavity using a dipole spectrometer magnet and a corresponding screen station in a dispersive section. For standard measurements, the gun is driven with an RF peak power of $\sim 6.6 \mathrm{MW}$ resulting in a maximum electric field gradient of about $60 \mathrm{MV} / \mathrm{m}$ at the cathode surface. The field at the MMMG phase as defined above is about $40 \mathrm{MV} / \mathrm{m}$. This yields a measured mean momentum of $6.68 \mathrm{MeV} / c$ with a spread of $10-20 \mathrm{keV} / \mathrm{c}$.

Electron beams exiting from the RF-gun still remain in the space charge dominated regime which the transverse emittance can increase while the beams are propagated along the beam transport line. In order to conserve low emittance beams a 9-cell normal conducting TESLA type RF cavity was used to achieve higher beam energy where space charge effects are reduced. This booster cavity was retained from the 2007 setup [11], but its position was shifted by $0.6 \mathrm{~m}$ downstream in order to fulfil the invariant envelope matching conditions for delivering the smallest projected emittance corresponding to a peak accelerating gradient of $60 \mathrm{MV} / \mathrm{m}$ at the photocathode. Standard operation conditions of the booster cavity for a bunch charge of $1 \mathrm{nC}$ were $10 \mathrm{~Hz}$ repetition rate, $50 \mu \mathrm{s}$ R pulse length and about $2.5 \mathrm{MW} \mathrm{RF}$ peak power, resulting in a final momentum of $\sim 14.8 \mathrm{MeV} / c$.

The performance of the booster cavity was strongly limited by the available cooling system. For the operation with a bunch charge lower than $1 \mathrm{nC}$, many micro-bunches in the train were used, which required a longer RF pulse length for the booster cavity and therefore forced a lower booster gradient. The booster gradient was adjusted to have a final momentum of $\sim 12.6 \mathrm{MeV} / c$ for emittance measurements of bunch charges below $1 \mathrm{nC}$. An RF pulse length of $100 \mu \mathrm{s}$ and RF peak power of about 1.3 MW were used.

\section{Emittance measurement method}

\subsection{Instruments and measurement procedure}

The transverse phase space distribution and the transverse projected emittance of the electron beam are measured using a single slit scan technique $[11,24]$. With this technique a spacecharge dominated electron beam is converted into a set of emittance dominated beamlets, which are used to measure the local beam divergence [25]. The Emittance Measurement SYstem (EMSY) consists of two orthogonal actuators for horizontal and vertical movements. On each actuator, a YAG or an OTR screen is mounted for measuring the beam transverse distribution. 10 - and $50-\mu \mathrm{m}$ opening slit masks are mounted on each actuator and are used to transversely cut the beam into thin slices. Stepping motors are employed to move and adjust the spatial position and angular orientation of the actuators with respect to the beam. A spatial position accuracy of about $1 \mu \mathrm{m}$ is obtained. The slit mask angle can be precisely adjusted for an optimum angular acceptance of the system with a precision of about 2 mrad per step.

Three EMSY stations were installed in the setup as shown in Fig. 1. The first emittance measurement system (EMSY1) behind the exit of the booster cavity is used for standard emittance measurements. It is located $5.74 \mathrm{~m}$ downstream the photocathode, because this position is the expected minimum emittance location obtained from beam dynamics simulations. A YAG powder-coated screen placed at $90^{\circ}$ to the beam axis with subsequent mirror at $45^{\circ}$ is used to measure the beam transverse size at EMSY1. A $10-\mu \mathrm{m}$ slit mask is used following a suggestion from detailed optimizations in Refs. [24,26], which combined the influence of the space-charge forces, the error from the initial beamlet transverse size, and the resolution in the measurement of 


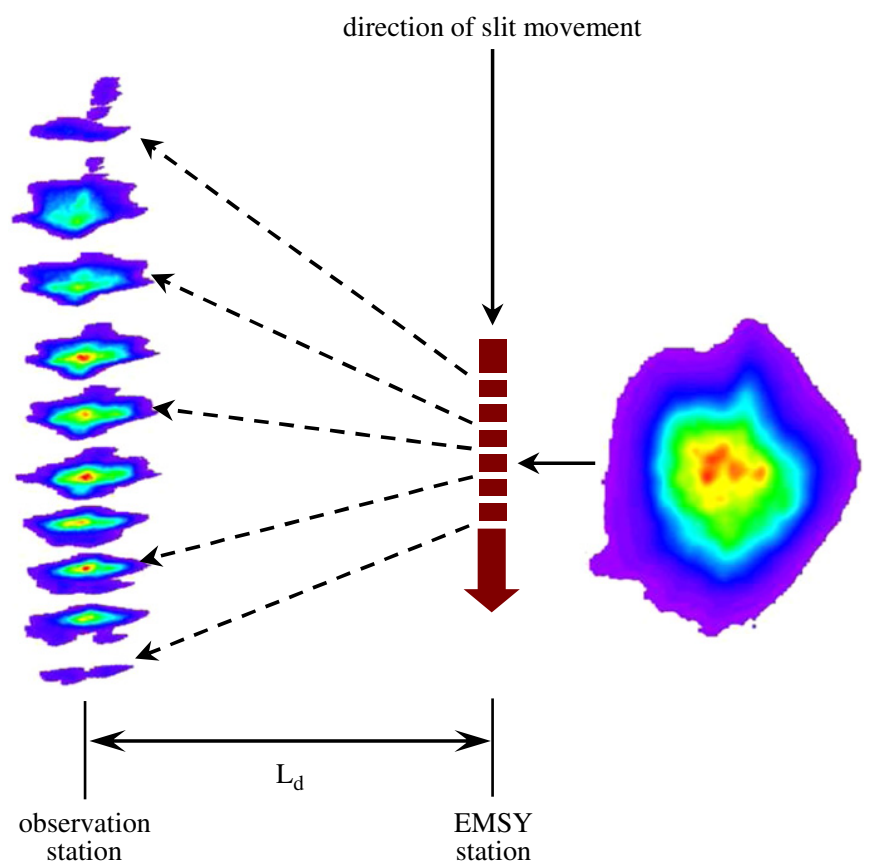

Fig. 5. Schematic picture showing the movement of a single slit to cut the beam transversely at an EMSY station. The beamlet images are observed on the YAG screen at the observation station at the distance $L_{d}$ downstream of the EMSY position (beam travels from right to left).

the beamlet divergence. The local divergence is estimated by measuring the profiles of the beamlets created by the slit on a YAG screen at an observation station with the distance $L_{d}$ downstream of the EMSY station as shown in Fig. 5. Also this screen is mounted at $90^{\circ}$ with respect to the beam axis and has a subsequent mirror at $45^{\circ}$. The distance between EMSY1 station and its beamlet observation screen is $2.64 \mathrm{~m}$.

For each set of emittance measurements, the transverse projected emittance is measured as a function of the main solenoid current. The solenoid current is varied within 10-15 A around the current which yields the minimum beam spot size at the EMSY station. The bucking solenoid current is set to compensate the residual magnetic field at the photocathode for each main solenoid current. Since the single slit scan technique is not a single shot measurement, uncertainties from the machine instability are included in the slit scan data.

In the emittance measurement procedure performed at PITZ prior to the run period in 2008-2009, a single slit scan was carried out with $200 \mu \mathrm{m}$ step size for each solenoid current, corresponding to about 20-30 beamlets for each transverse plane. At the optimum solenoid current, where the minimum emittance value was found, a fine slit scan was repeated with $50 \mu \mathrm{m}$ step size resulting in about 100 beamlets for each plane. The measurement time for a fine scan was $\sim 30 \mathrm{~min}$.

In the 2008-2009 run period, the emittance measurement procedure was improved to accelerate the slit scan and the data taking: the slit is continuously moved through the beam with constant speed within the scan limit. A typical speed is about $0.1-0.5 \mathrm{~mm}$ per second. The images at the beamlet observation screen are recorded with a CCD camera at $10 \mathrm{~Hz}$ repetition rate. The slit scan measurement and analysis are semiautomatically performed with the help of a client application called Emittance Measurement Wizard (EMWiz) [27]. The program combines an acquisition tool for performing slit scans and recording the beam spot or beamlet image and a postprocessing tool for analyzing the measured transverse phase space. The scan and data acquisition time for one measurement with a number of beamlets comparable to the old procedure is $\sim 20 \mathrm{~s}$ now. Since the measurement time is greatly reduced, several slit scans for a certain machine setting are now possible within a given time. This allows to distinguish between uncertainties of the measurement procedure and effects of jitter and drifts in the machine parameters.

In addition, more accurate criteria for the beamlet image quality using the full dynamic range of the CCD camera with 12 effective bits were defined. The number of laser pulses was adjusted to have a good beamlet intensity at the observation screen. This procedure includes checking pixel saturation during the scan and taking the statistics over all pixels in all beamlets. The measurement procedure was done in such a way that the low intensity tails of the measured phase-space distribution were included in the analysis in order to obtain a $100 \%$ rms emittance value.

\subsection{Emittance data analysis}

The single slit scan technique is used to measure the phase-space distribution of the electron beam at the slit position by measuring the projection of the beamlet images on the observation screen at some distance downstream of the slit location. As an example in Fig. 6 , the slit positions are plotted at the horizontal axis $(x)$ and the corresponding projection of the beamlet profiles characterizing the local divergence $x_{i}^{\prime}$ are binned as the vertical column at each slit position. The index $i$ corresponds to the specific slit position and runs from 1 to the total number of measured beamlets. This divergence value is related to the rms transverse momentum spread $\sqrt{\left\langle p_{x}^{2}\right\rangle}$ by $x^{\prime} \sim p_{x} /\left\langle p_{z}\right\rangle$ (if $\left\langle p_{z}\right\rangle \gg p_{x}$ ), where $\left\langle p_{z}\right\rangle$ is the mean longitudinal momentum.

The transverse emittance is proportional to the area of the phase-space ellipse and the normalized projected rms emittance can be calculated according to the following formula:

$\varepsilon_{n, x}=\beta \gamma \sqrt{\left\langle x^{2}\right\rangle\left\langle x^{\prime 2}\right\rangle-\left\langle x x^{\prime}\right\rangle^{2}}$.

The factor $\beta \gamma$ is defined from the measured beam momentum by using a spectrometer dipole magnet and a corresponding screen station in the dispersive section, where $\beta$ is the average electron velocity normalized to the speed of light and $\gamma$ is the relativistic Lorentz factor. The transverse rms beam size $\left(\sqrt{\left\langle x^{2}\right\rangle}\right)$ and the beam divergence $\left(\sqrt{\left\langle\boldsymbol{x}^{\prime 2}\right\rangle}\right)$ are obtained by analyzing the projection of the $\left(x, x^{\prime}\right)$ phase-space distribution from the slit scan

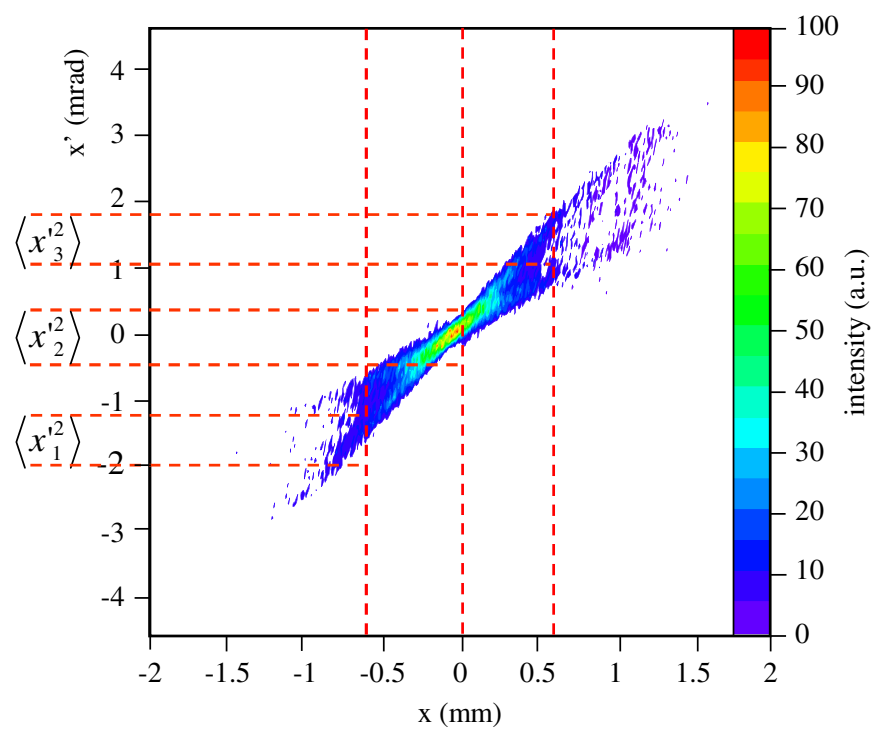

Fig. 6. Calculated transverse phase-space distribution obtained from the slit scan measurement. 
and $\left\langle x x^{\prime}\right\rangle$ is the covariance term where the relative position of the beamlet on the observation screen with respect to the corresponding slit position is taken into account.

From experimental experience, the measured transverse rms beam size from the measurement of beamlets at the observation screen (i.e. projection of the phase-space distribution in Fig. 6 on the horizontal axis) is smaller than the measured beam transverse size at the EMSY screen. This is caused by signal losses at the tails of the beam distribution due to the restricted sensitivity of the beamlet observation screen and the optical system. Losses in the tails of the phase-space distribution would result in an underestimation of the measured emittance value. To correct for low intensity signals in the tails of the phase-space distribution a correction factor $\sigma_{x} / \sqrt{\left\langle x^{2}\right\rangle}$ is introduced. It is determined as the ratio of the total rms beam size measured with the full beam on the EMSY screen where the slit scan is performed $\left(\sigma_{x}\right)$ and the beam size estimated from the phase space obtained from the slit scan on the observation screen $\sqrt{\left\langle x^{2}\right\rangle}$. Applying this correction factor to the standard normalized emittance formula in Eq. (1), we obtain the so-called corrected normalized projected emittance formula:

$\varepsilon_{n, x}=\beta \gamma \frac{\sigma_{x}}{\sqrt{\left\langle x^{2}\right\rangle}} \sqrt{\left\langle x^{2}\right\rangle\left\langle x^{\prime 2}\right\rangle-\left\langle x x^{\prime}\right\rangle^{2}}$.

This correction is identical the same as if the linear correlation is removed from the measured phase-space distribution and the resulting rms divergence is multiplied by the rms beam size $\sigma_{x}$ measured with the whole beam at the slit location. Emittance analysis using the formula in Eq. (2) results in a conservative emittance estimation because the correction factor $\sigma_{x} / \sqrt{\left\langle x^{2}\right\rangle}$ is almost always larger than 1 . For all measurements mentioned in this paper the correction factor value is between 0.9 and 2.6. The fact that the correction factor in some exceptional cases is smaller than 1 can be correlated to machine instabilities during the time when the slit is scanning over the full scan span and the beamlet images are taken. For the minimum emittance points in the solenoid scans for the four different charge levels $(1,0.5,0.25$, $0.1 \mathrm{nC})$ the correction factor is between 0.98 and 1.32 and its average is $1.12 \pm 0.04$. Since we have taken maximum effort to not cut any signal from the measured phase-space distribution in the analysis and we calculate real rms values (no fits are applied) we call our results " $100 \%$ rms values".

\section{Emittance optimization}

\subsection{Operation with bunch charge of $1 \mathrm{nC}$}

Measurements of the transverse projected emittance for a nominal bunch charge of $1 \mathrm{nC}$ were performed at the first emittance measurement system station (EMSY1), $5.74 \mathrm{~m}$ downstream the photocathode. In order to study the emittance dependence on the transverse laser size at the cathode, rms laser spot sizes of $0.26,0.29,0.38$ and $0.44 \mathrm{~mm}$ were applied. For each laser spot size single slit measurements were carried out as a function of the main solenoid current around the minimum beam size at the YAG screen of EMSY1. The gun RF phase was also varied to investigate its influence on the measured emittance. Experimental results showed that a gun phase of $+6^{\circ}$ from MMMG phase towards on-crest delivered the minimum measured emittance value. The field gradient at the photocathode at this phase is about $44 \mathrm{MV} / \mathrm{m}$. The measured mean momentum corresponding to this phase was $6.67 \mathrm{MeV} / c$ with a spread of about $40 \mathrm{keV} / c$. During these measurements the booster RF phase was set to be at the MMMG phase, while the gradient was set to the maximum accessible value allowed by the limit of the water cooling system. This results in a mean momentum of $14.7 \mathrm{MeV} / \mathrm{c}$ with a spread of about $150 \mathrm{keV} / c$.

The measured transverse projected emittance and rms beam size as a function of the main solenoid current for an rms laser spot size at the cathode of $0.38 \mathrm{~mm}$ and a gun RF phase of $+6^{\circ}$ from the phase of MMMG are shown in Fig. 6. The geometric mean emittance value $\left(\varepsilon_{n, x y}\right)$ is defined as $\varepsilon_{n, x y}=\sqrt{\varepsilon_{n, x} \varepsilon_{n, y}}$, where $\varepsilon_{n, x}$ and $\varepsilon_{n, y}$ are the horizontal and the vertical projected normalized rms emittance. In the following parts of this paper, the index " $n$ " will be suppressed for simplicity although only normalized quantities will be reported. The geometric mean rms laser spot size $\left(\sigma_{x y}\right)$ is derived from $\sigma_{x y}=\sqrt{\sigma_{x} \sigma_{y}}$, where $\sigma_{x}$ and $\sigma_{y}$ are the horizontal and the vertical rms beam size, respectively. As can be seen in Fig. 7, the minimum measured geometric mean emittance was obtained at a main solenoid current of 387 A corresponding to a solenoid peak field of $228 \mathrm{mT}$. Typically, the optimum main solenoid current, which delivers minimum measured emittance for a bunch charge of $1 \mathrm{nC}$, is found 2-5 A above the focusing point, it is $3 \mathrm{~A}$ in this case. At this minimum, a geometric mean emittance was measured to be $0.99 \mathrm{~mm}$ mrad.

Statistical measurements for the same machine setting but with an improved beam steering condition through the booster cavity were performed at the optimum main solenoid current (387 A) obtained from the solenoid scan measurement. The minimum measured normalized rms emittance was $0.72 \pm 0.01 \mathrm{~mm}$ mrad in the horizontal plane and $1.09 \pm 0.02 \mathrm{~mm}$ mrad in the vertical plane. This corresponds to a geometric mean emittance of $0.89 \pm$ $0.01 \mathrm{~mm}$ mrad. The statistical uncertainty given in the results were obtained from four measurement sets which were taken in a time period of $\sim 30 \mathrm{~min}$. However, this small emittance value could not reproducibly be measured in the next shift due to the instabilities of the machine, e.g. gun RF phase. Detailed studies concerning this issue are discussed in Section 4.4. Reconstructed phase-space distributions from one set of the statistical measurements are shown in Fig. 9 for both transverse directions. For sufficient beamlet signal intensity, 27 and 17 electron bunches were used for the measurements of the horizontal and vertical phase space, respectively. A summary of machine and beam parameters for the operation conditions that lead to the smallest measured emittance for the nominal bunch charge of $1 \mathrm{nC}$ are listed in Table 1 .

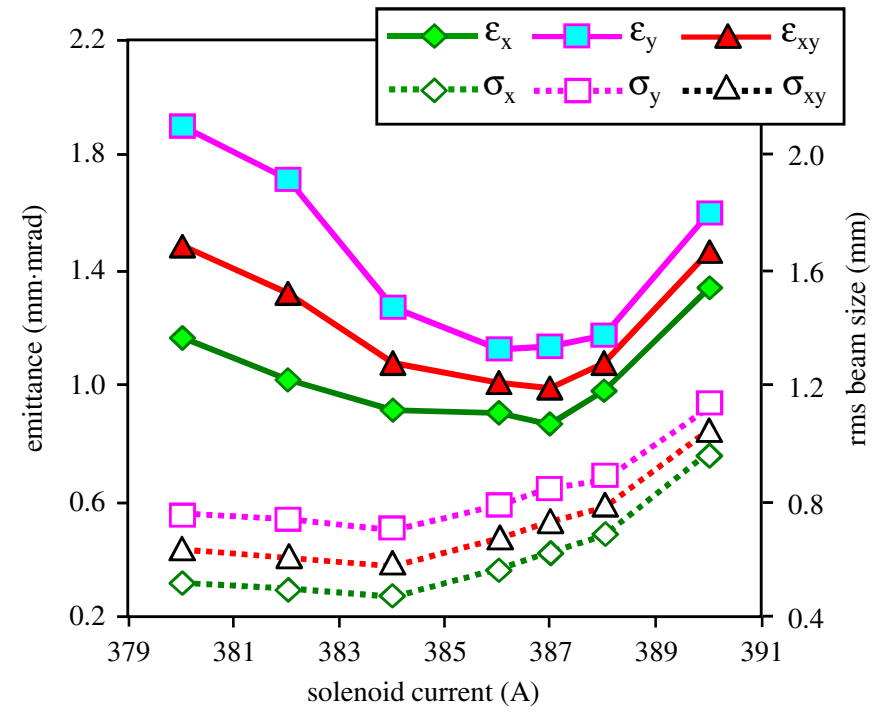

Fig. 7. Measured normalized projected emittance (left axis) and rms beam size (right axis) as a function of main solenoid current for a measured rms laser spot size at the cathode of $0.38 \mathrm{~mm}$. The minimum emittance was obtained at a main solenoid current of 387 A corresponding to the solenoid peak field of $228 \mathrm{mT}$. 
Table 1

Optimum machine and beam parameters and measured results to obtain the minimum normalized transverse projected emittance of the electron beam with a bunch charge of $1 \mathrm{nC}$. The RF phases of the gun and the booster cavity are defined with respect to the phase of MMMG. The phase with positive sign $(+)$ is towards on-crest.

\begin{tabular}{ll}
\hline Parameter & Value \\
\hline Geometrical rms laser spot size & $0.38 \mathrm{~mm}$ \\
Laser temporal length (FWHM) & $23.1 \mathrm{ps}$ \\
Laser temporal rise-/fall-time & $2.1 / 2.4 \mathrm{ps}$ \\
Gun RF phase & $+6^{\circ}$ \\
Booster RF phase & $0^{\circ}$ \\
Beam momentum after the gun & $6.67 \mathrm{MeV} / \mathrm{c}$ \\
Final beam momentum & $14.8 \mathrm{MeV} / \mathrm{c}$ \\
Main solenoid current & $387 \mathrm{~A}$ \\
Geometric mean rms beam spot size & $0.60 \mathrm{~mm}$ \\
Horizontal emittance & $0.72 \pm 0.01 \mathrm{~mm} \mathrm{mrad}$ \\
Vertical emittance & $1.09 \pm 0.02 \mathrm{~mm} \mathrm{mrad}$ \\
Geometric mean emittance & $0.89 \pm 0.01 \mathrm{~mm} \mathrm{mrad}$ \\
\hline
\end{tabular}
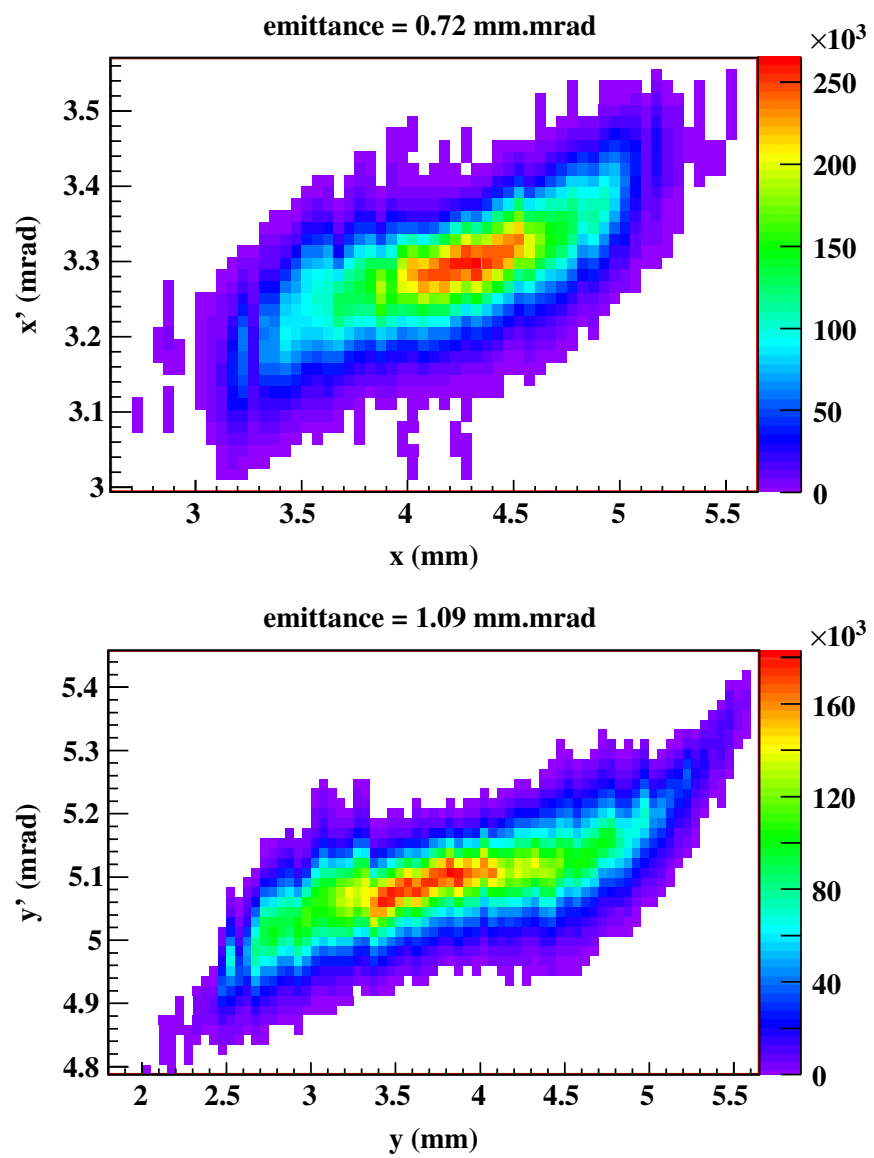

Fig. 8. Measured horizontal (top) and vertical (bottom) phase-space distributions for $100 \%$ charge intensity for a bunch charge of $1 \mathrm{nC}$. The measurements were performed at a beam momentum of $14.7 \mathrm{MeV} / \mathrm{c}$, main solenoid current of $387 \mathrm{~A}$ gun RF phase of $+6^{\circ}$ from the MMMG phase towards on-crest and booster phase at maximum acceleration condition.

Results of emittance optimization for different laser spot sizes for fixed gradients and RF phases of the gun and the booster cavity are shown in Fig. 9. The minimum emittance was obtained for an rms laser spot size of $0.38 \mathrm{~mm}$. As can be seen in Figs. 7-9 there is an asymmetry in the measured horizontal and vertical emittance values. One possible reason is an asymmetric shape of the booster cavity at the RF waveguide input location. Asymmetric electromagnetic fields due to an opening in the vertical wall of the booster cavity can introduce a vertical kick to the

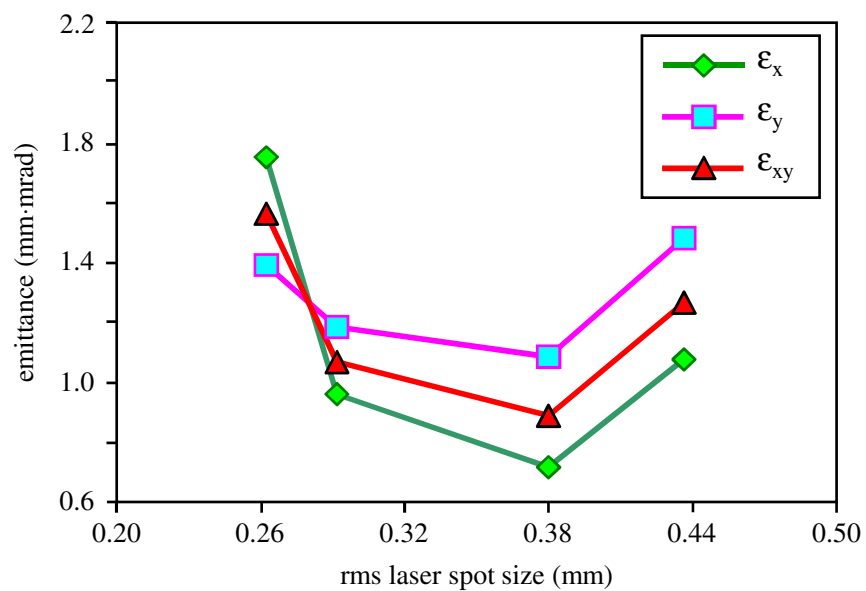

Fig. 9. Measured normalized projected emittance as a function of the rms laser spot size at the cathode for a bunch charge of $1 \mathrm{nC}$. Each data point was obtained from the optimization with the solenoid scan. The gun and the booster RF phases are $+6^{\circ}$ and $0^{\circ}$ from the phase of MMMG towards on-crest, correspondingly.

beams. This in general leads to a bigger beam size in the vertical plane and consequently to a bigger emittance. This is not the case for a small rms laser spot size $(0.26 \mathrm{~mm})$, pointing to an effect of high space charge intensity at the cathode during charge emission for small laser spot size at the cathode. It is important to be noted here that the transverse shape of the photocathode laser spot and the beam after exiting the RF-gun were round. The beams with asymmetric and tilted transverse shape were observed after the booster acceleration.

\subsection{Operation with bunch charges of $0.5,0.25$ and $0.1 \mathrm{nC}$}

Optimization of the transverse projected emittance was also performed for electron bunch charges of $0.5,0.25$, and $0.1 \mathrm{nC}$. Gun and booster $\mathrm{RF}$ phases of $+6^{\circ}$ and $0^{\circ}$ from the phase of MMMG towards on-crest were used. The gun gradient was set to be at the maximum available value of $\sim 60 \mathrm{MV} / \mathrm{m}$ at the photocathode. Due to the lower charge, more laser pulses were used to adjust the intensity of the beamlet signal at the observation screen. This required a longer RF pulse length for the gun and the booster cavity. In the measurements for these three bunch charges the booster cavity was operated with an RF pulse length of $100 \mu \mathrm{s}$. Since a limitation in the capacity of the water cooling system restricted the operation of the booster cavity with long RF pulse trains at high acceleration, a lower booster gradient was used and resulted in a final beam momentum of about $12.6 \mathrm{MeV} / c$.

The measurements were performed with the same procedure and at the same measurement location as in the case of $1 \mathrm{nC}$. The dependence of the transverse projected emittance on the rms laser spot size at the cathode was also studied. Similar to the case of $1 \mathrm{nC}$, the vertical emittance value were measured to be larger than the horizontal ones. This relation is inverted when the rms laser spot size is smaller than $0.26,0.21$ and $0.15 \mathrm{~mm}$ for a charge of $0.5,0.25$ and $0.1 \mathrm{nC}$, respectively. The possible reason for this phenomenon was discussed in Section 4.1. The measured geometric mean emittance as a function of the rms laser spot size at the cathode for all four bunch charges are shown together in Fig. 10. These emittance values were analyzed from the measured phase-space distributions, where $100 \%$ of the detected signal was used. The minimum measured emittance values are $0.89,0.64$, 0.47 , and $0.33 \mathrm{~mm}$ mrad for a bunch charge of $1,0.5,0.25$ and $0.1 \mathrm{nC}$, respectively. These minimum points were obtained at the rms laser spot sizes of $0.38,0.26,0.21$ and $0.15 \mathrm{~mm}$ at the cathode. 


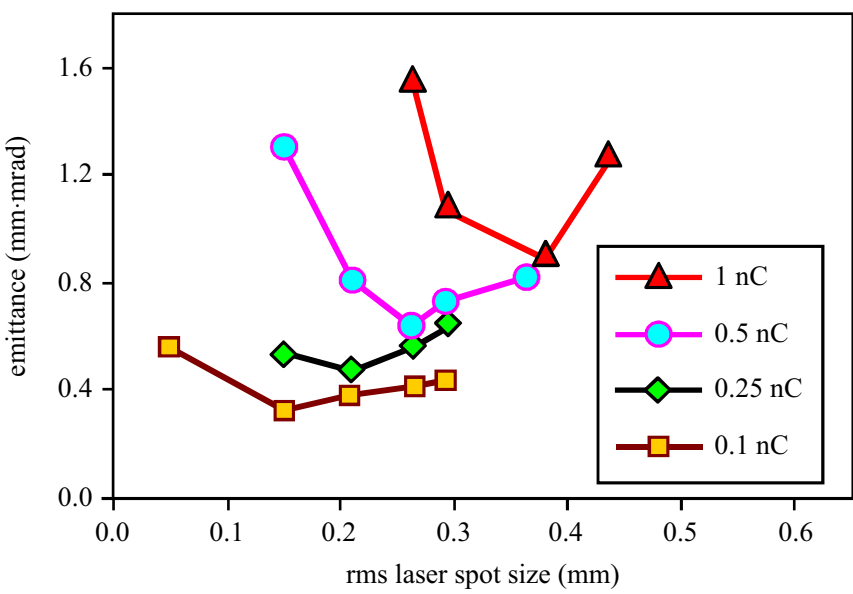

Fig. 10. Measured geometric mean emittance $\left(\varepsilon_{x y}\right)$ as a function of the rms laser spot size at the cathode for a charge of $1,0.5,0.25$ and $0.1 \mathrm{nC}$ per bunch. The gun phase was at $+6^{\circ}$ from the phase of MMMG towards on-crest. The booster phase was kept at the maximum acceleration phase. For each data point the solenoid current was varied to deliver the minimum emittance value.

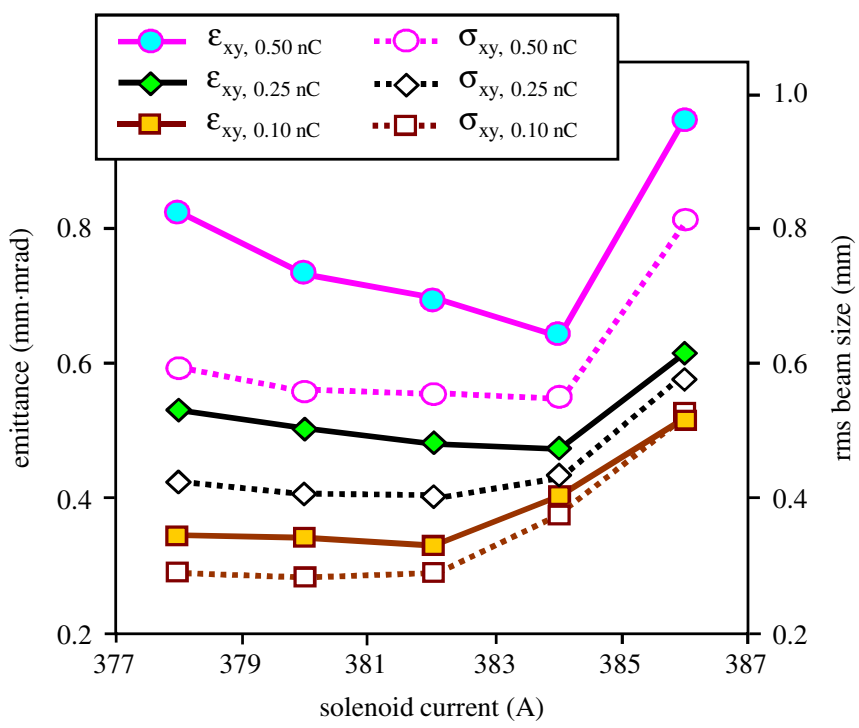

Fig. 11. Measured normalized projected emittance (left axis) and rms beam size (right axis) as a function of main solenoid currents for a bunch charge of $0.5,0.25$ and $0.1 \mathrm{nC}$. The optimum rms laser spot sizes at the cathode are $0.26,0.21$ and $0.15 \mathrm{~mm}$, respectively.

The solenoid scan for each laser spot size was also performed. The measured geometric mean emittance and the rms beam size as a function of main solenoid current for the optimum rms laser spot sizes at the cathode for the three bunch charges are shown in Fig. 11. The solenoid currents which deliver the minimum measured emittances were found at $384 \mathrm{~A}$ for a bunch charge of 0.5 and $0.25 \mathrm{nC}$ and at $382 \mathrm{~A}$ for a charge of $0.1 \mathrm{nC}$. The minimum measured beam sizes were obtained at the solenoid currents of 384, 382 and $380 \mathrm{~A}$, respectively. Machine and beam conditions used in the measurements of a bunch charge of $0.5,0.25$ and $0.1 \mathrm{nC}$ are listed in Table 2.

\subsection{Emittance vs. charge cut}

Since the particles in the low intensity tails of the transverse phase-space distribution do not contribute to the lasing process in short wavelength FELs but have a significant contribution to the $100 \%$ rms emittance they can be subsequently removed in the analysis procedure in order to obtain a kind of core emittance
Table 2

Optimum machine and beam parameters and measured results to obtain the minimum projected emittance for a bunch charge of $0.5,0.25$ and $0.1 \mathrm{nC}$. The quantities $\sigma_{l}, \tau, t_{r}, t_{f}, I_{\text {main }}$, and $\sigma_{x y}$ represent the rms laser spot size, the laser temporal FWHM length, the laser temporal rise-time, the laser temporal fall-time, the main solenoid current, and the geometric mean rms beam size, respectively, where $\varepsilon_{x}, \varepsilon_{y}$, and $\varepsilon_{x y}$ are the horizontal, the vertical, and the geometric mean emittances. The gun RF phase was at $+6^{\circ}$ from the phase of MMMG towards oncrest. The booster phase was kept at the maximum acceleration condition and the beam momentum was $12.6 \mathrm{MeV} / \mathrm{c}$.

\begin{tabular}{lccc}
\hline Parameter & $0.5 \mathrm{nC}$ & $0.25 \mathrm{nC}$ & $0.1 \mathrm{nC}$ \\
\hline$\sigma_{l}(\mathrm{~mm})$ & 0.26 & 0.21 & 0.15 \\
$\tau(\mathrm{ps})$ & 23.6 & 23.7 & 24.9 \\
$t_{r}(\mathrm{ps})$ & 2.5 & 2.4 & 2.8 \\
$t_{f}(\mathrm{ps})$ & 3.5 & 3.1 & 4.6 \\
$I_{\text {main }}(\mathrm{A})$ & 384 & 384 & 382 \\
$\sigma_{x y}(\mathrm{~mm})$ & 0.55 & 0.43 & 0.29 \\
$\varepsilon_{x}(\mathrm{~mm} \mathrm{mrad})$ & 0.53 & 0.42 & 0.29 \\
$\varepsilon_{y}(\mathrm{~mm} \mathrm{mrad})$ & 0.77 & 0.53 & 0.37 \\
$\varepsilon_{x y}(\mathrm{~mm}$ mrad) & 0.64 & 0.47 & 0.33 \\
\hline
\end{tabular}

which is important for the FEL process. In addition the emittance as a function of the charge cut can be used to compare the PITZ results with other facilities where in general intrinsic cuts in the tails of the measured phase-space distributions are performed, e.g. by omitting the beamlets in the tails of the beam distribution when performing pepper pot method or by fitting Gaussian distributions to the measurement during quadrupole scans.

A cut on the low intensity tails of the measured phase-space distribution to estimate the emittance as a function of the charge cut was performed. As an example the measured phase space distribution for $1 \mathrm{nC}$ bunch charge was estimated by removing $10 \%$ of the total bunch charge from the lowest density regions in the measured phase-space distributions in Fig. 8 and the $90 \%$ charge intensity phase-space distributions are shown in Fig. 12. The result shows that a geometric mean emittance as small as $0.67 \mathrm{~mm}$ mrad is achieved.

The phase-space distribution of the minimum geometric mean emittance results are re-analyzed as a function of the charge cut in the tails of the phase-space distribution. The results for the bunch charges of 1, 0.5, 0.25 and $0.1 \mathrm{nC}$ are illustrated in Fig. 13. An example of one of the interesting cases is for $0.25 \mathrm{nC}$ with $5 \%$ charge cut, where a normalized projected emittance of about $0.4 \mathrm{~mm}$ mrad is obtained. This emittance value is comparable to the value obtained at LCLS [28], where an FEL operation with a wavelength of $0.15 \mathrm{~nm}$ was demonstrated.

\subsection{Stability of emittance measurement}

During the emittance measurements, strong fluctuations of the gun RF phase at a given position within the RF pulse train were observed [29]. Therefore, stability measurements were performed for the nominal bunch charge of $1 \mathrm{nC}$ with the machine conditions and beam characteristics listed in Table 3.

Machine and beam parameters in Table 3 are about the same as in the case of minimum emittance except the laser pulse length was about 4 ps shorter as well as the rise and fall time were longer due to the damage of some crystals in the laser pulse shaper. This resulted in emittance values larger than for the optimum case as discussed in Section 4.1. A standard deviation of the geometric mean emittance fluctuation of about 5\% (rms) was observed for a measurement time of about $3 \mathrm{~h}$ (Fig. 14). The rms beam size jitter was measured to be about $4 \%$. Long term stability measurements were performed over 4 days and the results are shown in Fig. 15. The rms deviation of the horizontal, vertical and geometric mean emittance is $6 \%, 8 \%$ and $5 \%$, respectively. 

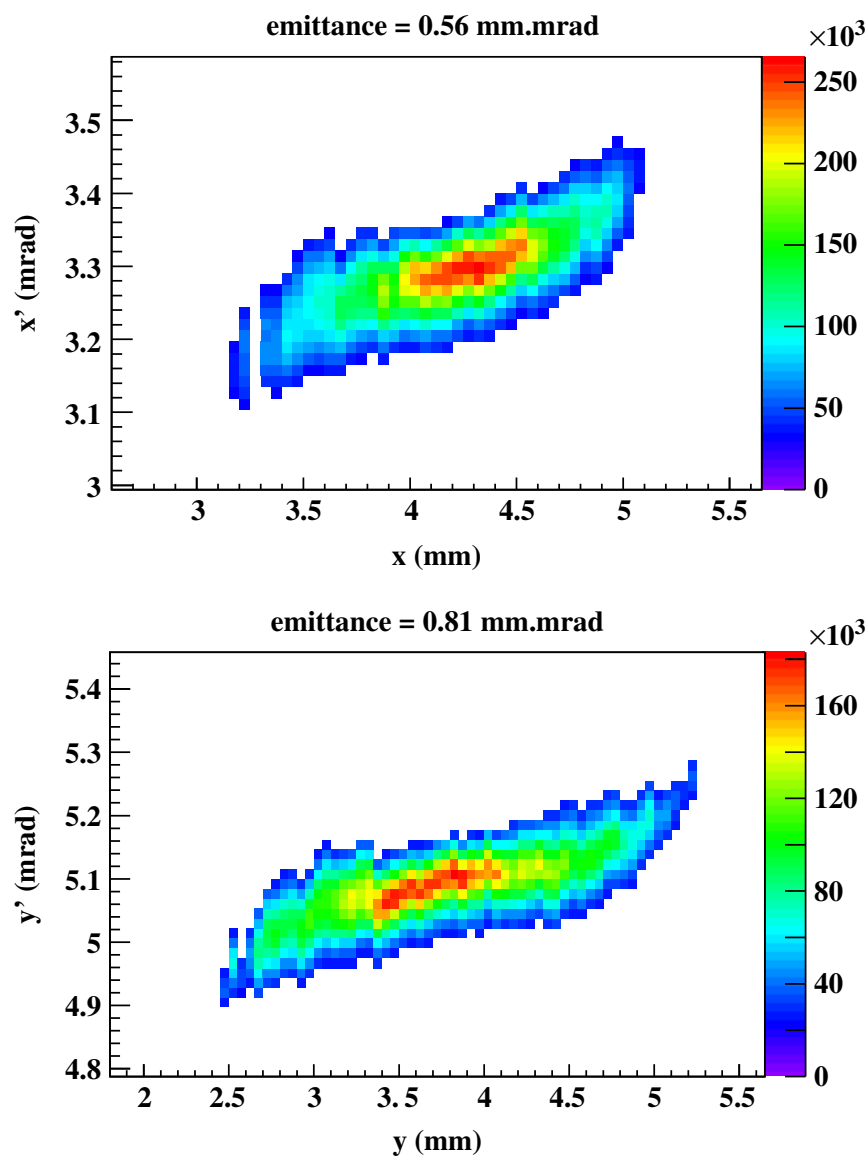

Fig. 12. Measured horizontal (top) and vertical (bottom) phase-space distributions for $90 \%$ charge intensity for a bunch charge of $1 \mathrm{nC}$. A geometric mean emittance $\left(\varepsilon_{x y}\right)$ of $0.67 \mathrm{~mm}$ mrad is obtained. Machine conditions and beam characteristics are the same as in the measurements of the phase space distributions in Fig. 8.

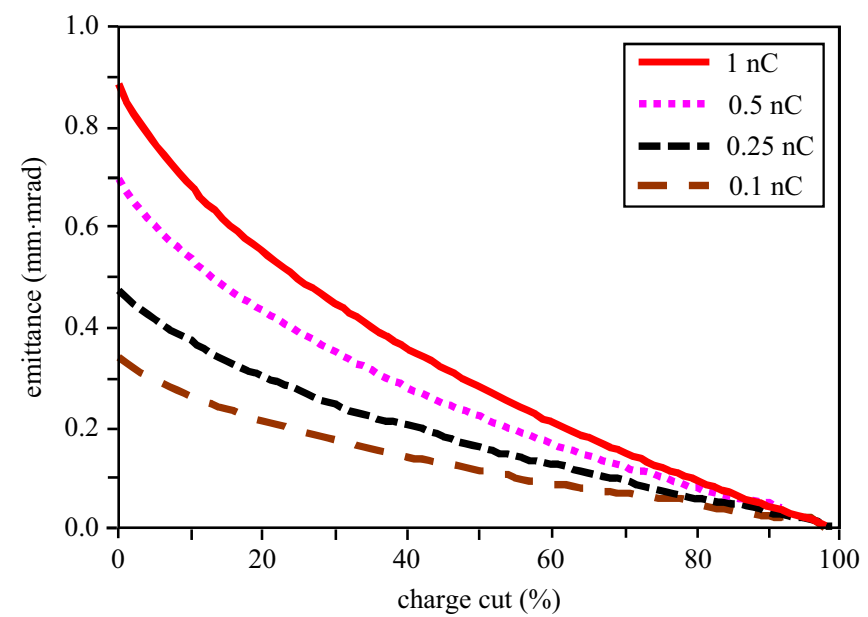

Fig. 13. Minimum geometric mean emittance $\left(\varepsilon_{x y}\right)$ as a function of a charge cut starting from the lowest density tails in the phase-space distribution for a bunch charge of $1,0.5,0.25$ and $0.1 \mathrm{nC}$. The $100 \%$ emittance values reported here are for a charge cut $0 \%$ (no charge cut).

\section{Comparison of measurements with simulations}

Beam dynamics simulations using the space-charge particle tracking code ASTRA [30] were performed to study the dependence of the emittance on the laser spot size at the cathode as
Table 3

Summary of the main parameters for stability measurements shown in Figs. 13 and 14 . The gun and the booster RF phases were at $+6^{\circ}$ and $0^{\circ}$ from the MMMC phase towards on-crest.

\begin{tabular}{ll}
\hline Parameter & Value \\
\hline Charge per bunch & $1 \mathrm{nC}$ \\
Laser pulse length (FWHM) & $18.9 \mathrm{ps}$ \\
Laser temporal rise-/fall-time & $3.0 / 3.2 \mathrm{ps}$ \\
Laser rms spot size at the cathode & $0.38 \mathrm{~mm}$ \\
Mean momentum & $14.4 \mathrm{MeV} / \mathrm{c}$ \\
Main solenoid current & $384 \mathrm{~A}$
\end{tabular}

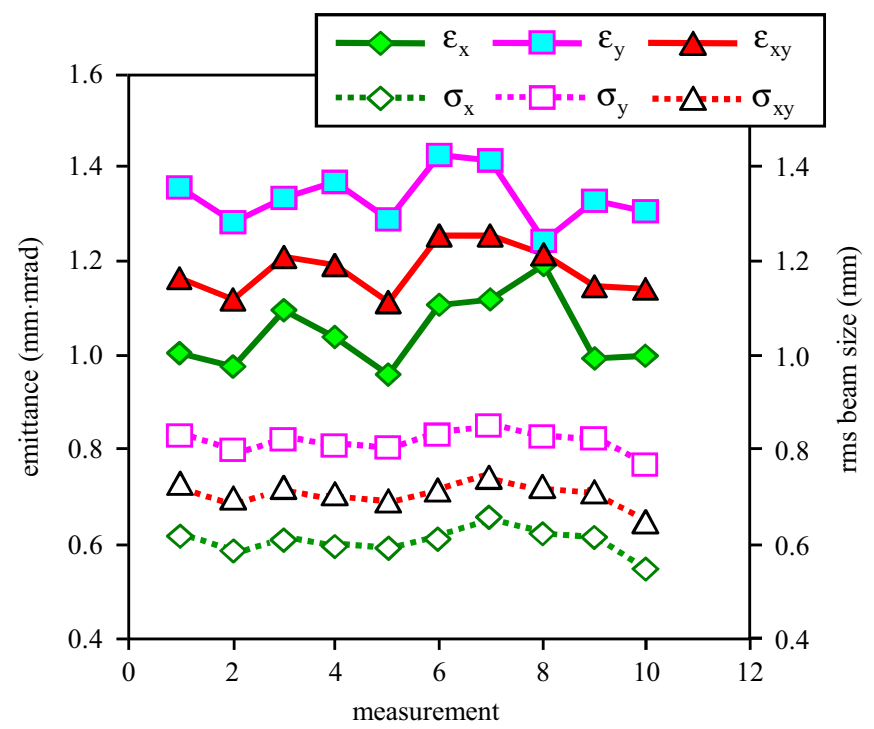

Fig. 14. Stability check of the measured emittance (left axis) and rms beam size (right axis) for the measurement time of about $3 \mathrm{~h}$. The rms standard deviation of the geometric mean emittance and the beam size are $5 \%$ and $4 \%$, respectively. The measurements were performed for a bunch charge of $1 \mathrm{nC}$ with the parameters in Table 3.

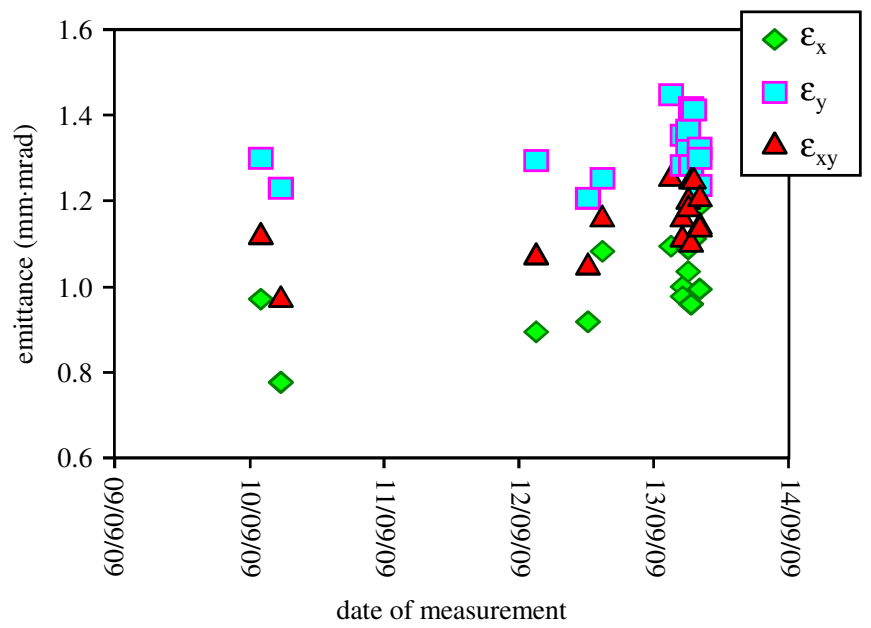

Fig. 15. Fluctuations of the measured emittance for an operation time over 4 days. The measurements were performed for a bunch charge of $1 \mathrm{nC}$ with the parameters in Table 3. The rms standard deviation of the fluctuation of the geometric mean emittance is $\sim 5 \%$.

well as gun phase, solenoid magnetic field, booster phase and booster gradient. Simulation studies included machine and beam parameters according to the experimental conditions. The laser flat-top temporal length as well as the rise and fall time was set to 
be the measured values for each bunch charge as shown in Tables 1 and 2. The simulated accelerating gradients at the cathode and in the booster cavity were adjusted to achieve the same maximum mean momenta as in the measurements. The gun and the booster phases are defined such that zero degree corresponds to the phase of MMMG.

As an example, a series of simulations were performed to find the optimum parameters for the case of an electron beam with a bunch charge of $1 \mathrm{nC}$. A temporal laser profile of flat-top shape with 23 ps FWHM pulse length and 2 ps rise and fall time, as in the measured temporal profile in Fig. 2, was used. An RF-gun field ratio (cathode to center of the full-cell) of 1.05, as in the measured field profile [17], was applied. Twenty thousand macro-particles were tracked in the ASTRA simulations, and 60 radial grid ring and 100 longitudinal grid were used for the space charge calculation. A theoretical initial electron kinetic energy of $0.55 \mathrm{eV}$ were applied [31]. Some approximations were included in the simulations discussed in this paper. An additional emitted charge and a modification of the bunch due to Schottky-like effects is not taken into account. The transverse laser distribution was assumed to be uniform and not to be modified by inhomogeneous cathode emission.

The simulated mean momentum and momentum spread as a function of the maximum accelerating gradient at the cathode are shown in Fig. 16. The solenoid field was chosen to be $226.2 \mathrm{mT}$, which allows all electrons to be extracted and accelerated out of the gun cavity with good focusing. The results in Fig. 16 suggest that a maximum gradient at the photocathode of $58.6 \mathrm{MV} / \mathrm{m}$ delivers an electron bunch with a mean momentum of $6.68 \mathrm{MeV} / \mathrm{c}$ at the MMMG phase as in the measurement. In the next step, the final mean momentum was tuned by varying the booster gradient and the result shows that in order to achieve a final beam momentum of $14.8 \mathrm{MeV} / \mathrm{c}$ at the MMMG phase as in the measurement a booster gradient of $15 \mathrm{MV} / \mathrm{m}$ should be used. By following the simulation results, we used the gun and the booster gradients of $58.6 \mathrm{MV} / \mathrm{m}$ and $15 \mathrm{MV} / \mathrm{m}$, respectively, in all simulations for a bunch charge of $1 \mathrm{nC}$.

The dependence of the projected emittance on the gun RF phase was studied with results shown in Fig. 17. The rms laser spot size at the cathode used in this simulation was $0.41 \mathrm{~mm}$. The booster RF phase was kept at the maximum acceleration phase. The solenoid peak field was varied to obtain a minimum emittance value for each gun phase. The result in Fig. 17 suggests that the smallest emittance value is obtained with the gun phase of $+0.5^{\circ}$ from the phase of MMMG towards on-crest whereas $+6^{\circ}$

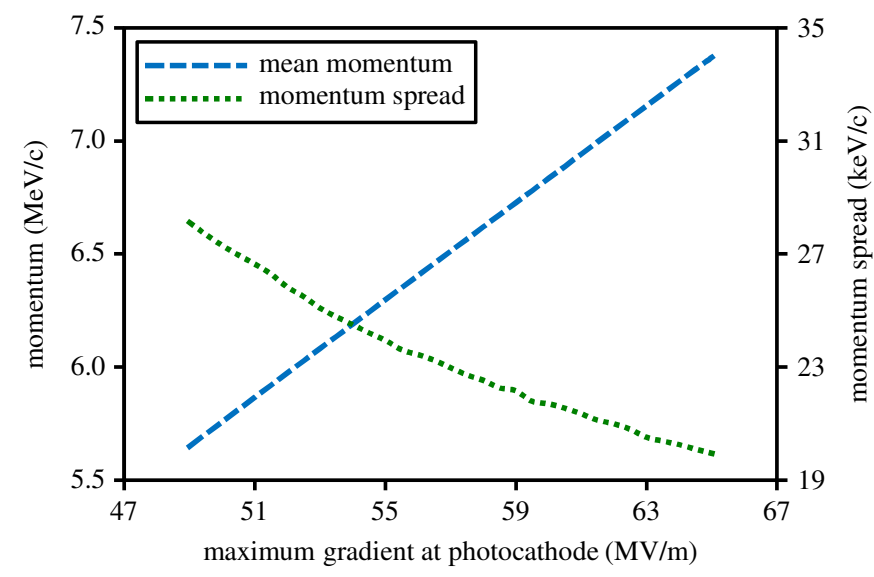

Fig. 16. Simulated mean momentum (left axis) and momentum spread (right axis) as a function of the maximum accelerating gradient at the photocathode for a bunch charge of $1 \mathrm{nC}$. The gun phase is at the phase of MMMG. The solenoid peak field is $226.2 \mathrm{mT}$. The simulations were conducted for a position $2.8 \mathrm{~m}$ downstream of the photocathode.

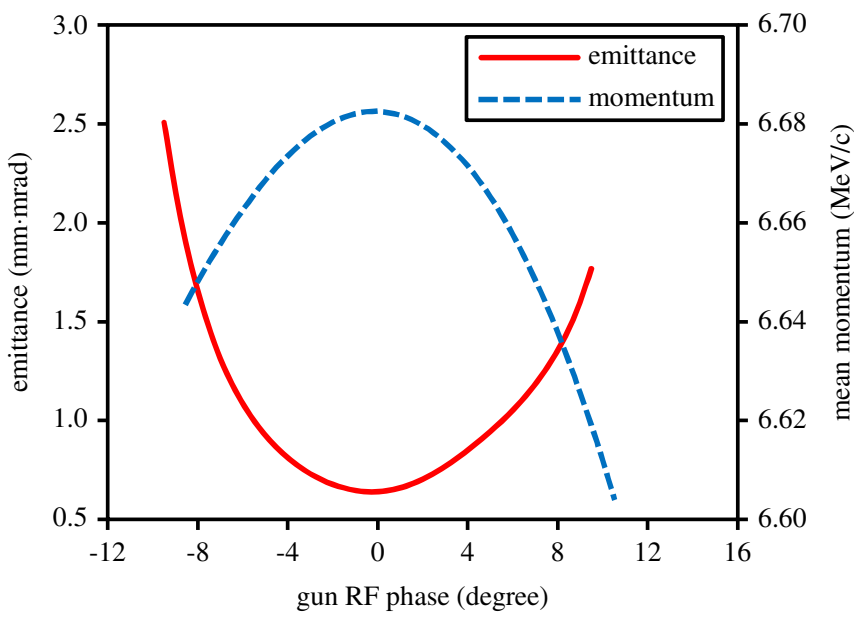

Fig. 17. Simulated dependence of emittance (left axis) and mean momentum of the electron bunch after the gun cavity (right axis) on the gun RF phase for an electron bunch of $1 \mathrm{nC}$ with a final momentum of $14.8 \mathrm{MeV} / \mathrm{c}$. The $0^{\circ}$ phase is referred to as the phase of MMMG. The rms laser spot size at the cathode is $0.41 \mathrm{~mm}$. The booster phase was set at the maximum acceleration and the solenoid peak field was optimized to deliver the minimum emittance for each gun phase.

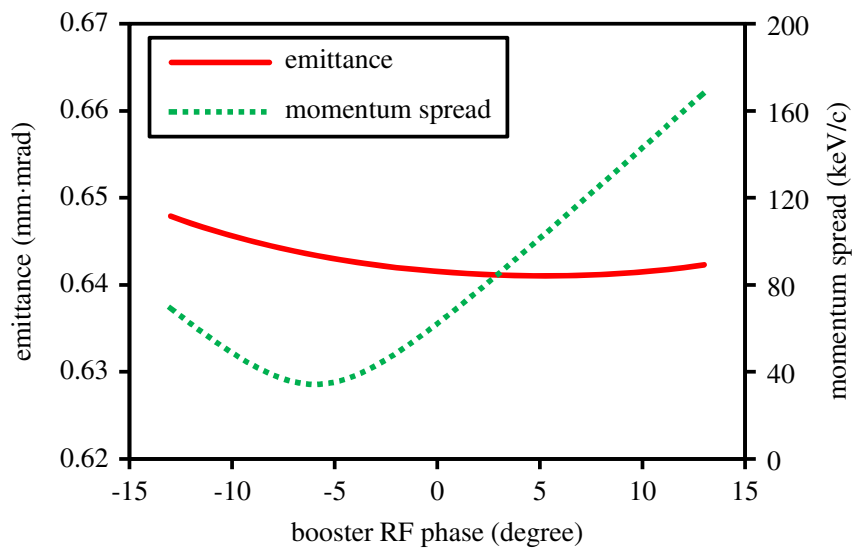

Fig. 18. Simulated normalized projected emittance (left axis) and momentum spread (right axis) as a function of booster RF phase for a bunch charge of $1 \mathrm{nC}$ at $5.74 \mathrm{~m}$ downstream of the photocathode (EMSY1 position). The zero phase is defined with respect to the phase of MMMG. The rms laser spot size at the cathode is $0.41 \mathrm{~mm}$ and the gun phase is $+0.5^{\circ}$ from the MMMG phase towards on-crest. The solenoid peak field was optimized to deliver the minimum emittance at each booster phase.

was experimentally found. At the gun phase of $+0.5^{\circ}$ the field gradient at the photocathode is $40 \mathrm{MV} / \mathrm{m}$.

Similar to the simulations of the gun RF phase, the emittance dependence on the booster RF phase was investigated and the results are illustrated in Fig. 18. The gun phase was set to be at $+0.5^{\circ}$ from the MMMG phase and the solenoid field was scanned to deliver the minimum emittance value. Simulation results show that the minimum projected emittance is found at a booster RF phase of $+5^{\circ}$ from the phase of MMMG towards on-crest. However, at this phase the correlated momentum spread increases from $62 \mathrm{keV} / \mathrm{c}$ to $101 \mathrm{keV} / \mathrm{c}$. Since steering magnets are needed to be used in the PITZ beamline in order to transport the beam through the center of different measurement devices the larger momentum spread will lead to larger spot sizes and consequently larger emittance values. Therefore a beam with smaller momentum spread is preferable for the photo-injector. The results depicted in Fig. 18 show that the projected emittance depends only weakly on the booster phase around the MMMG phase $\left(0^{\circ}\right)$, which was used in the measurement cases. The emittance value is only $0.08 \%$ smaller when the booster phase is at $+5^{\circ}$ from the MMMG phase but the momentum spread 
becomes significantly larger by $63.2 \%$. In all simulations (Figs. 16-18), the optimum rms laser spot size at the cathode of $0.41 \mathrm{~mm}$ was used (see next paragraph).

The dependence of the emittance on the rms laser spot size at the cathode was also studied. Simulation predictions in Fig. 19 show that an rms laser spot size at the cathode of $0.41 \mathrm{~mm}$ delivers the minimum emittance value of $0.64 \mathrm{~mm}$ mrad at the considered emittance measurement station, EMSY1 $(5.74 \mathrm{~m}$ downstream the photocathode). Unfortunately, the same laser spot size could not be produced experimentally due to the limitation of available apertures in the laser system in the described setup. However, the emittance value increases only $1.4-2.0 \%$ for the experimentally available rms laser spot sizes of $30 \mu \mathrm{m}$ smaller and larger than $0.41 \mathrm{~mm}$. The emittance measurement result for a bunch charge of $1 \mathrm{nC}$ shows that the minimum measured projected emittance was found at an rms laser spot size of $0.38 \mathrm{~mm}$, which is close to the expectation from the simulation.

The simulated dependence of the emittance on the peak solenoid field is shown in Fig. 20. In this simulation, the optimum

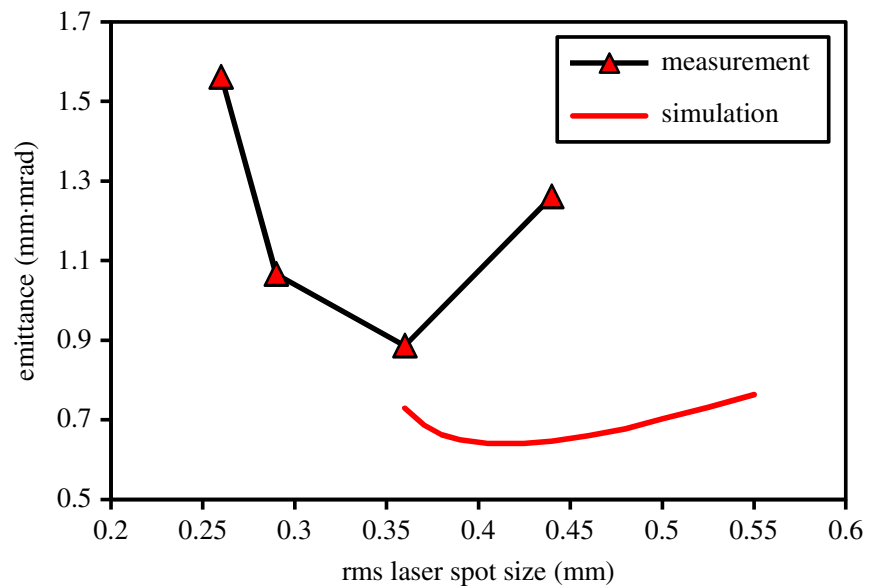

Fig. 19. Simulated dependence of the emittance at the EMSY1 station $(5.74 \mathrm{~m}$ downstream of the photocathode) on the rms laser spot size at the cathode for a charge of $1 \mathrm{nC}$ per bunch. The gun phase and the solenoid field were optimized to obtain the minimum emittance value for each laser spot size. The booster phase was set to be at the MMMG phase. The optimum rms laser spot size at the cathode of $0.41 \mathrm{~mm}$ delivers the minimum emittance value of $0.64 \mathrm{~mm}$ mrad.

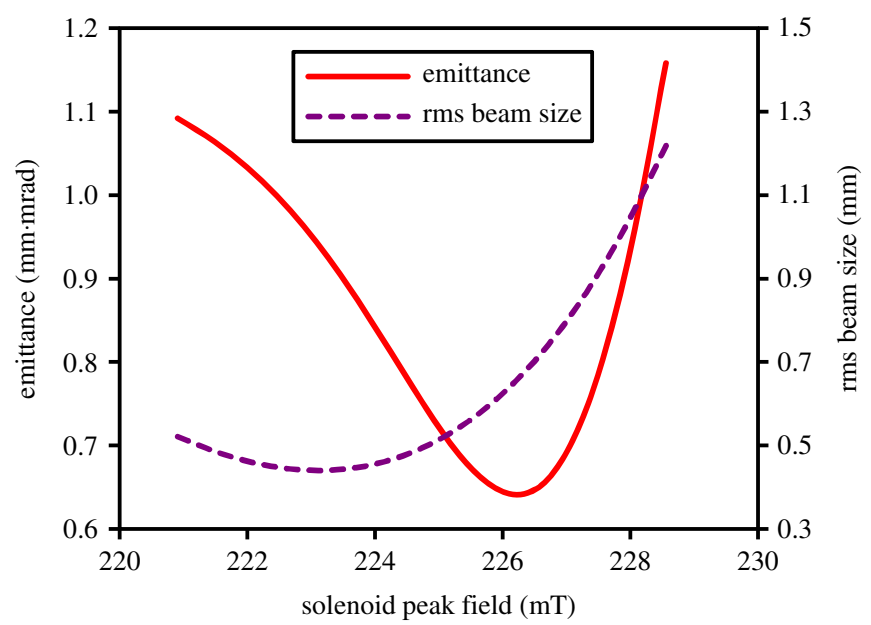

Fig. 20. Simulated dependence of the emittance (left axis) and the rms beam size (right axis) on the solenoid peak field for a bunch charge of $1 \mathrm{nC}$ with the final mean momentum of $14.8 \mathrm{MeV} / c$. The rms laser spot size at the cathode was optimized to be $0.41 \mathrm{~mm}$. The RF phases of the gun and the booster were set to be at $+0.5^{\circ}$ and $0^{\circ}$ from the MMMG phase. The simulations were performed for a position $5.74 \mathrm{~m}$ downstream of the photocathode (EMSY1 station). rms laser spot size of $0.41 \mathrm{~mm}$ was used, the gun and the booster $\mathrm{RF}$ phases were at $+0.5^{\circ}$ and $0^{\circ}$ from the phase of MMMG. The optimization results show that the minimum emittance value was obtained with a peak solenoid field of $226.2 \mathrm{mT}$. This solenoid field corresponds to a main solenoid current of $384 \mathrm{~A}$, whereas the smallest beam size is obtained at $379 \mathrm{~A}$. In the measurement, the minimum emittance point was obtained at a main solenoid current of $387 \mathrm{~A}$, which means there is a discrepancy of $3 \mathrm{~A}$ (corresponding to a solenoid peak field of $2 \mathrm{mT}$ ) between the measurement and the simulation. A part of the discrepancy can be related to a possible error in the magnetic field calibration of the solenoid magnets.

The dependence of the emittance on the booster cavity position and gradient were simulated in order to study the emittance conservation using a post-acceleration cavity. In both simulations, a gun phase of $+0.5^{\circ}$ from the MMMG phase and the rms laser spot size at the cathode of $0.41 \mathrm{~mm}$, which yield the minimum projected emittance value, were used. The booster RF phase was set at the phase of MMMG. The simulations show that the minimum emittance can be conserved by locating a booster cavity at the position of the beam envelope waist. It means that the starting point of the RF field of the TESLA booster cavity should be at $3.64 \mathrm{~m}$ downstream the photocathode. According to this simulation result, the position of the booster cavity was then shifted by $0.6 \mathrm{~m}$ downstream as mentioned in Section 2.3. Simulations of the projected emittance and the momentum of the electron beam as a function of the booster gradient were performed and the results are shown in Fig. 21. The solenoid peak field was scanned to obtain the minimum emittance value for each simulation point. It is clearly seen that higher booster gradients deliver smaller projected emittance values. However, the rate of emittance reduction slows down for larger booster gradients.

Fig. 22 shows the simulated evolution of the normalized projected emittance and the rms beam size along the beam line (z-position). The minimum normalized projected emittance at the position of the EMSY1 station, $5.74 \mathrm{~m}$ downstream the photocathode, is expected to be $0.64 \mathrm{~mm}$ mrad. Machine and beam parameters used in the beam dynamics simulations to define this minimum emittance are summarized in Table 4 . The emittance value stays almost constant for $z$-positions between about 5.5 and $6.1 \mathrm{~m}$. Then, it increases almost linearly proportional to the longitudinal distance. This fact shows that the mean momentum

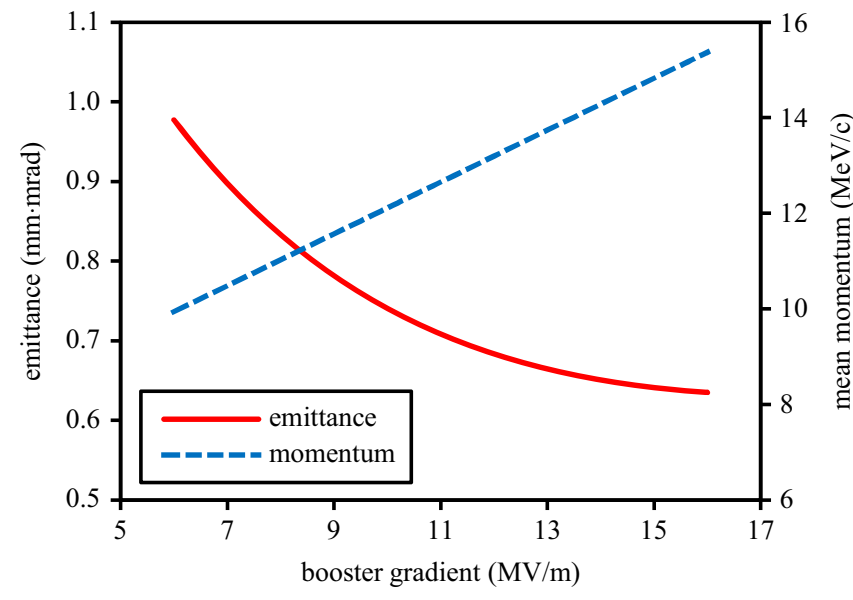

Fig. 21. Simulated normalized projected emittance (left axis) and mean momentum (right axis) as a function of booster gradient for a bunch charge of $1 \mathrm{nC}$. The gun and the booster RF phases are at $+0.5^{\circ}$ and $0^{\circ}$ from the phase of MMMG. The simulations were conducted for a position $5.74 \mathrm{~m}$ downstream of the photocathode (EMSY1 location). 


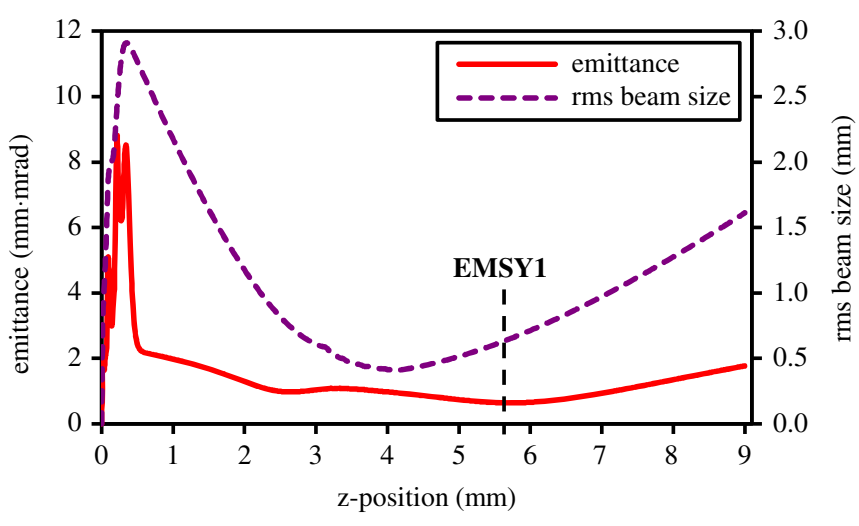

Fig. 22. Simulated evolution of the normalized transverse projected emittance (left axis) and the rms beam size (right axis) along the PITZ beam line described with the parameters in Table 4. The position of the emittance measurement station (EMSY1) at $5.74 \mathrm{~m}$ downstream the photocathode is indicated. The simulated normalized projected emittance at the EMSY1 station is $0.64 \mathrm{~mm}$ mrad.

Table 4

Optimum main parameters for the PITZ beam used in the beam dynamics simulations for the bunch charge of $1 \mathrm{nC}$. The gun and the booster RF phases are $+0.5^{\circ}$ and $0^{\circ}$ from the phase of MMMG, respectively.

\begin{tabular}{ll}
\hline Parameter & Value \\
\hline Transverse rms laser size & $0.41 \mathrm{~mm}$ \\
Laser FWHM pulse length & $23 \mathrm{ps}$ \\
Laser rise-/fall-time & $2 / 2 \mathrm{ps}$ \\
Peak accelerating field at the cathode & $58.6 \mathrm{MV} / \mathrm{m}$ \\
Peak accelerating field in the booster & $15 \mathrm{MV} / \mathrm{m}$ \\
Beam mean momentum after the gun & $6.68 \mathrm{MeV} / \mathrm{c}$ \\
Beam mean momentum after the booster & $14.8 \mathrm{MeV} / \mathrm{c}$ \\
Solenoid peak field & $226.2 \mathrm{mT}$ \\
\hline
\end{tabular}

of $14.8 \mathrm{MeV} / \mathrm{c}$ is not enough for suppressing the space-charge force. A higher final beam momentum by using a higher gradient or a longer accelerating cavity is thus required in order to conserve the transverse projected emittance.

Simulation studies of the influence of different machine and beam parameters on the emittance were also performed for the bunch charges of $0.5,0.25$ and $0.1 \mathrm{nC}$. The maximum accelerating gradient at the cathode of $58.6 \mathrm{MeV} / c$ was applied in order to obtain a beam mean momentum of $6.68 \mathrm{MeV} / \mathrm{c}$ at the MMMG phase after the gun acceleration. The simulated accelerating gradient of the booster cavity was adjusted to be $10.4 \mathrm{MV} / \mathrm{m}$ to deliver a final mean momentum of $12.3 \mathrm{MeV} / c$, which was used in the measurements. According to simulation results in Fig. 19 the emittance value increases by $13.3 \%$ when reducing the booster gradient from $15 \mathrm{MV} / \mathrm{m}$ (used for the $1 \mathrm{nC}$ case) to this value $10.4 \mathrm{MV} / \mathrm{m}$.

The rms laser spot sizes at the cathode in these simulations included the values resulting from available beam shaping apertures used in the measurements. In case of small laser spot sizes at the cathode the space charge effect dominates the electron emission process. Simulations of the emitted charge per bunch and the projected emittance as a function of the laser spot size at the cathode were performed for the desired bunch charges of $1,0.5,0.25$ and $0.1 \mathrm{nC}$ and the results are shown in Figs. 23 and 24. The Schottky-like effects were not included in the simulations. The results reveal that by considering only the photoemission process due to laser pulses hitting the cathode, the desired bunch charge of $1,0.5,0.25$ and $0.1 \mathrm{nC}$ cannot be produced by using an rms laser spot size smaller than $0.36,0.24,0.15$ and $0.10 \mathrm{~mm}$, respectively.

Simulation results in Fig. 24 suggest that the minimum emittance values should be found at an rms laser spot size of $0.41,0.29,0.21$ and $0.12 \mathrm{~mm}$ for a bunch charge of $1,0.5,0.25$ and

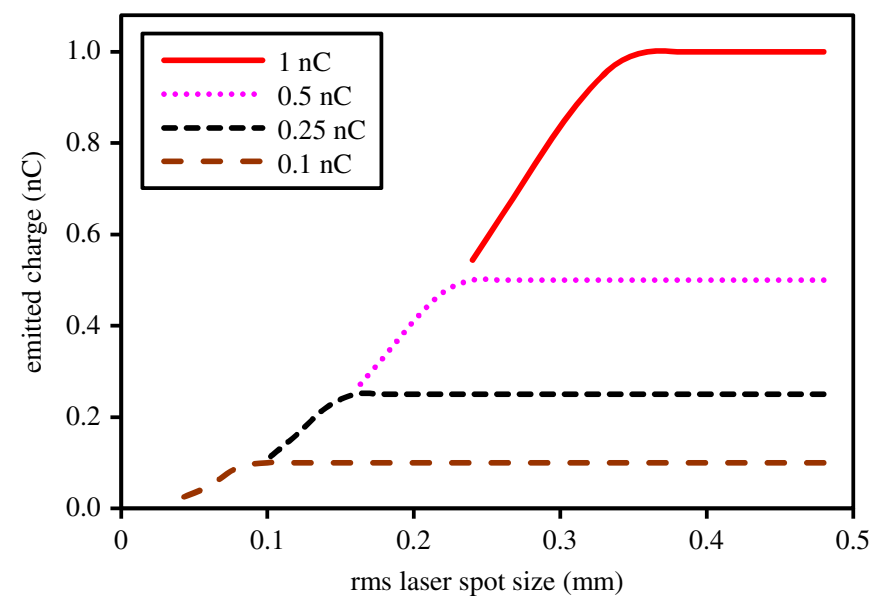

Fig. 23. Simulated emitted electron bunch charge for different rms laser spot sizes at the cathode for a bunch charge of $1,0.5,0.25$ and $0.1 \mathrm{nC}$. The gun RF phase was set to be at the phase of MMMG phase. The solenoid peak field was chosen to have good focusing for the electron bunch exiting the RF-gun cavity.

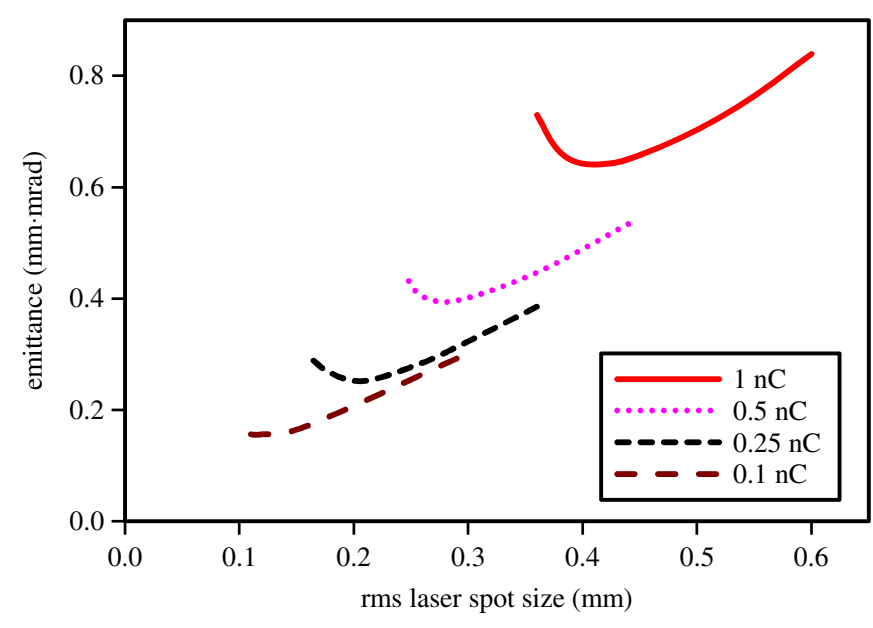

Fig. 24. Simulated normalized projected emittance as a function of the rms laser spot size for a bunch charge of $1,0.5,0.25$ and $0.1 \mathrm{nC}$. For each data point, the gun $\mathrm{RF}$ phase and the solenoid peak field were varied to deliver the minimum emittance value. The booster RF phase was kept at MMMG condition. The laser flat-top FWHM as well as the temporal rise- and fall-time were adjusted to be the same as the measurement conditions in Tables 1 and 2. The final mean momentum is $14.8 \mathrm{MeV} / \mathrm{c}$ for the bunch charge of $1 \mathrm{nC}$ and $12.3 \mathrm{MeV} / \mathrm{c}$ for 0.5 , 0.25 and $0.1 \mathrm{nC}$.

$0.1 \mathrm{nC}$, respectively. Unfortunately, the same laser spot size could not be produced in the experiment due to the limitation of available apertures in the measurement cases. Further investigation will be performed in the future upgraded PITZ setup using a remote control of diaphragm beam shaping aperture enabling fine tuning of the aperture size. A summary of the measured and simulated optimum rms laser spot sizes at the cathode for minimum transverse projected emittance values are shown in Table 5.

Measured and simulated normalized projected emittances as a function of the bunch charge are shown in Fig. 25. In the simulations, rms laser spot sizes at the cathode of $0.38,0.26$, 0.21 and $0.15 \mathrm{~mm}$ were used for the bunch charges of $1,0.5,0.25$ and $0.1 \mathrm{nC}$, respectively. In the simulation case, the gun RF phase was optimized to deliver minimum projected emittance for each bunch charge. The measured main solenoid current and the simulated peak solenoid field were varied to yield the smallest emittance points. In the measurement case, the emittance value of each point is the geometric mean of the normalized projected 
Table 5

Measured and simulated optimum rms laser spot sizes at the cathode and minimum normalized transverse projected emittances for the bunch charges of $1,0.5,0.25$ and $0.1 \mathrm{nC}$, where $\sigma_{m}$ is the measured rms laser spot size, $\sigma_{s}$ is the simulated rms laser spot size, $\varepsilon_{x y}$ is the measured geometric mean emittance, and $\varepsilon$ is the simulated emittance.

\begin{tabular}{lllll}
\hline Parameter & $1 \mathrm{nC}$ & $0.5 \mathrm{nC}$ & $0.25 \mathrm{nC}$ & $0.1 \mathrm{nC}$ \\
\hline$\sigma_{m}(\mathrm{~mm})$ & 0.38 & 0.26 & 0.21 & 0.15 \\
$\sigma_{s}(\mathrm{~mm})$ & 0.41 & 0.29 & 0.21 & 0.12 \\
$\varepsilon_{x y}(\mathrm{~mm}$ mrad $)$ & 0.89 & 0.64 & 0.47 & 0.33 \\
$\varepsilon(\mathrm{mm} \mathrm{mrad})$ & 0.64 & 0.40 & 0.25 & 0.16 \\
\hline
\end{tabular}

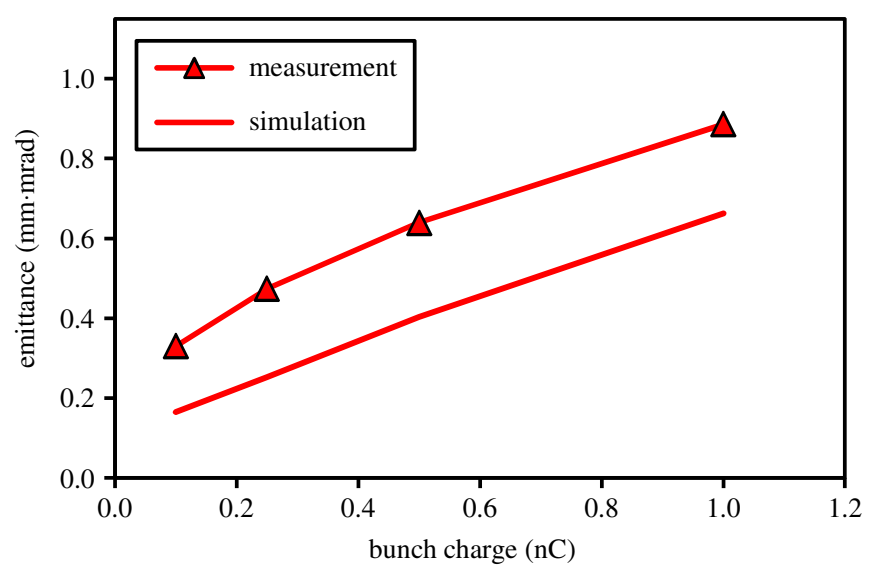

Fig. 25. Simulated and measured normalized projected emittance as a function of the bunch charge. In the measurement case, the value of each data point is the geometric mean emittance for both transverse planes. Machine and beam conditions are described in the text.

emittance for the two transverse planes. The gun RF phase during the measurements was $+6^{\circ}$ from the phase of MMMG towards on-crest. In both measurement and simulation, the booster RF phase of MMMG was used.

Comparison of measurement results with the results from beam dynamics simulations shows that the measured projected emittances for the four bunch charges are larger than the simulation values. One part of the mismatch could be due to a large fluctuation of the RF-gun phase for a given bunch in the train and over the pulse train. This instability is considered to be the source of fluctuations of the measured electron beam size and emittance [29]. These fluctuations can result in a jitter of beam position, beam momentum and phase space distribution. Furthermore the emittance measurement using a single slit scan technique is not a single shot measurement and the procedure even involves pulse trains of varying lengths for the beamlet intensity integration. Therefore the gun phase jittering will cause a smearing of the phase space distributions and results in larger measured emittances. The gun RF phase jitter also results in an uncertainty of the optimum gun phase condition.

Simulations of the influence of a gun phase jitter on the beam size and emittance were performed by overlapping the phase space distributions for different gun phases around the minimum emittance phase. Then, the rms beam size and the emittance were calculated from the overlapped distributions. The simulation results for a bunch charge of $1 \mathrm{nC}$ are presented in Fig. 26 and show that emittance and beam size increase significantly for larger gun phase jitter. For a phase jitter of $\pm 6^{\circ}$ peak-to-peak, the emittance increases by $30.5 \%$, corresponding to an emittance value of $0.84 \mathrm{~mm}$ mrad, which is close to the value obtained from the measurement $(0.89 \mathrm{~mm}$ mrad).

Stabilization of the RF-gun phase shot-to-shot and over a long RF pulse train was difficult to achieve with the described RF-gun

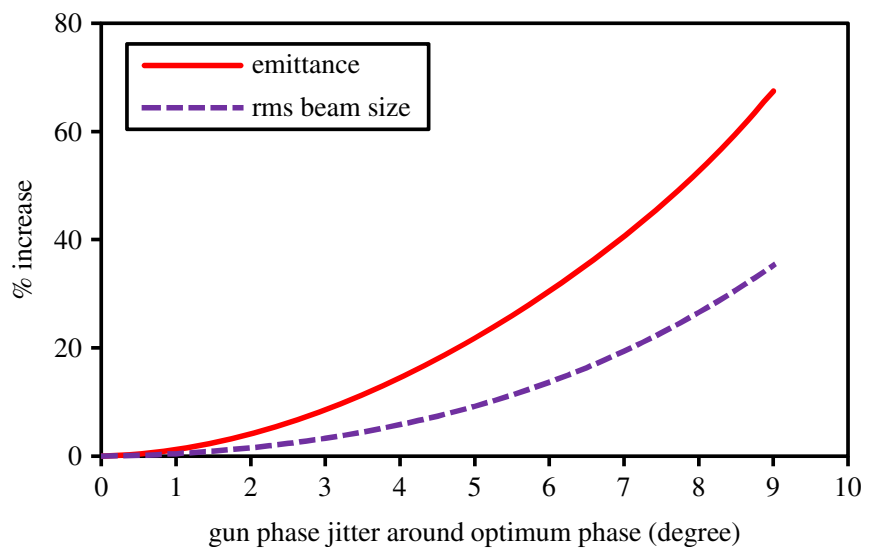

Fig. 26. Simulated emittance and beam size increase due to gun phase jittering (peak-to-peak) for a charge of $1 \mathrm{nC}$ per bunch. Machine and beam parameters used in this simulation are the same as for the simulation in Fig. 15.

system. The RF wave was supplied by a 10-MW multibeam klystron via two equal waveguide arms, where each arm contained a directional coupler for measuring forward and reflected RF waves to control the feeding of the RF waves to the gun. A ceramic vacuum window was installed after each directional coupler. Then, a T-shape combiner was used to mix both RF waves and feed them into the gun cavity. Due to the absence of a directional coupler downstream of the T-combiner, the low level RF regulation could only use the vector sum built from forward and reflected RF powers measured in both arms. A working RF feedback was not available, therefore only feed forward was used. The possibility of cross-talk of signals from both directional couplers through the T-combiner under not welldefined resonance conditions of the gun cavity caused complications of the low level RF regulation and consequently large measured gun phase fluctuations between $10^{\circ}$ and $15^{\circ}$ (peak-to-peak) [29]. The jitter of the RF-gun phase complicates not only the emittance measurement but also the procedure for the machine setup, resulting in uncertainty in the definition of the gun RF phase and an increase in the measured emittance value.

A second source of discrepancy could be due to the thermal emittance contribution. The experimentally estimated thermal emittance is about 1.5 times higher than the value expected from theory [32]. Therefore, the value of the initial kinetic energy of $0.55 \mathrm{eV}$ assumed in the ASTRA simulations might be too small. Nevertheless the thermal emittance measurement is difficult and has a large error bar. A third source of the difference is probably due to the missing knowledge concerning the booster cavity field under the high RF power operation which was used in the simulations. Another source of discrepancy could happen for small laser spot sizes (as shown in Section 5) where the space charge dominates the electron emission process and Schottky-like effects play a significant role. This results in a charge production enhancement due to the presence of a high electric field at the cathode. The electric fields distort the potential barrier at the cathode surface resulting in a lower work function and a higher quantum efficiency of the cathode material. This effect can lead to a difference between the optimum laser spot size obtained from the measured and the simulated cases.

\section{Conclusion and outlook}

This paper described and discussed the optimization of the projected transverse emittance for the RF-gun prototype 4.2 and the machine setup at the photo-injector test facility at DESY, location Zeuthen (PITZ), in the run period 2008-2009. This gun cavity was operated with an accelerating gradient of about 
$60 \mathrm{MV} / \mathrm{m}$ at the photocathode. A successful demonstration of the required $R F$ parameters for the injector of the European XFEL was performed with an average RF power of $50 \mathrm{~kW}$ for a $700 \mu$ s long RF pulse of $7.1 \mathrm{MW}$ peak power at $10 \mathrm{~Hz}$ repetition rate. The gun has been delivered to FLASH and is then in continuous operation since February 2010 [33].

The emittance of electron beams produced by using flat-top temporal laser pulses was measured for bunch charges of $1,0.5$, 0.25 and $0.1 \mathrm{nC}$. Small emittance values beyond the requirements of the European XFEL were achieved, especially for $1 \mathrm{nC}$ bunch charge. A minimum rms geometric average normalized projected emittance of two transverse directions of $0.89 \pm 0.01 \mathrm{~mm}$ mrad was measured at $1 \mathrm{nC}$ bunch charge. This value is obtained from the $100 \% \mathrm{rms}$ phase-space distribution. If $10 \%$ of the charge is removed from the lowest density regions in the phase-space distribution, an rms normalized projected emittance of $0.67 \mathrm{~mm}$ mrad is achieved. Emittance optimizations for bunch charges below $1 \mathrm{nC}$ were also performed. The $100 \%$ rms geometric mean emittance of $0.64,0.47$ and $0.33 \mathrm{~mm}$ mrad were measured for the bunch charges of $0.5,0.25$ and $0.1 \mathrm{nC}$, respectively.

Beam dynamics simulations based on the measured machine conditions and beam characteristics were carried out. Some discrepancies of the measured values from the simulation results were observed. The RF-gun phase instabilities are considered to be the major source of the mismatch between measurement and simulation results. Further investigations will be continued in the next run period using newly installed RF-gun and booster cavities [34]. The gun phase stability is anticipated to be improved for the new gun cavity with a new 10-MW in-vacuum directional coupler installed downstream the T-combiner. Direct monitoring and control of the combined forward and reflected RF waves will allow an RF feedback control loop for the amplitude and phase stabilization in the new setup. A new Cut Disk Structure booster cavity will allow to accelerate the electrons to above $20 \mathrm{MeV}$ with high stability and will be suitable for operation with long pulse trains due to an efficient cooling system. In addition, the field profile of this booster cavity is well known. Altogether these should lead to an improvement in the simulation knowledge and a more flexible booster operation.

\section{Acknowledgments}

This work was partly supported by the European Community, contracts RII3-CT-2004-506008 and 011935, and by the 'Impuls- und Vernetzungsfonds' of the Helmholtz Association, contract VH-FZ-005.

The authors would like to thank R. Brinkmann, U. Gensch, C. Pagani, J. Rossbach, W. Sandner, and I. Tsakov for continuous support of the PITZ project. We would like to acknowledge K. Flöttmann, D. Reschke, A. Brinkmann and J. Ziegler who have worked on the dryice sublimation-impulse cleaning of the RF gun cavity. We would also like to express our sincere gratitude and appreciation to all colleagues of the PITZ collaboration for contributions to the upgrade and commissioning of the PITZ accelerator and beam diagnostic components. Special thanks go to M. Großand A. Oppelt for proofreading this manuscript and providing comments.

\section{References}

[1] W. Ackermann, et al., Nature Photonics 1 (2007) 336

[2] M. Altarelli, et al., European XFEL Technical Design Report, DESY 2006-097, DESY, Hamburg, 2007.

[3] J. Arthur, et al., LCLS Conceptual Design Report, SLAC-R-593, SLAC, Stanford, 2002.

[4] T. Tanaka, T. Shintake (Eds.), SCSS X-FEL Conceptual Design Report, RIKEN Harima Institute/Spring-8, Mikazuki, Japan, 2005.

[5] C.J. Bocchetta, et al., Conceptual Design Report for the FERMI@Elettra Project, Sincrotrone trieste, 2007.

[6] R. Ganter (Ed.), SwissFEL Conceptual Design Report, PSI Bericht 10-04, PSI, 2010.

[7] P. Schmüser, M. Dohlus, J. Rossbach, Ultraviolet and Soft X-Ray Free-Electron Lasers: Introduction to Physical Principles, Experimental Results, Technological Challenges, Springer-Verlag, Berlin, Heidelberg, 2008.

[8] M. Cornacchia, P. Emma, Transverse to Longitudinal Emittance Exchange, SLAC-PUB-9225, SLAC, Stanford, 2002.

[9] P. Emma, et al., Physical Review Special Topics Accelerators and Beams 9 (2006) 100702.

[10] K. Kim, A. Sessler, Transverse-longitudinal phase-space manipulations and correlations, beam cooling and related topics,. in: International Workshop on Beam Cooling and Related Topics, AIP Conference Proceedings, vol. 821, 2006, pp. $115-138$.

[11] F. Stephan, et al., Physical Review Special Topics Accelerators and Beams 13 (2010) 020704

[12] C. Boulware, et al., in: Proceedings of the 30th International Free Electron Laser Conference, Gyeongju, South Korea, 2008, paper THAAU05.

[13] G. Asova, et al., in: Proceedings of the 1st International Particle Accelerator Conference, Kyoto, Japan, 2010, paper TUPE010.

[14] S. Lederer, et al., in: Proceedings of the 23rd Particle Accelerator Conference, Vancouver, Canada, 2009, paper MO6RFP055.

[15] L. Monaco, et al., in: Proceedings of the 23rd Particle Accelerator Conference, Vancouver, Canada, 2009, paper MO6RFP072.

[16] TTF Photocathodes Database 〈http://wwwlasa.mi.infn.it/ttfcathodes/〉.

[17] S. Rimjaem, et al., in: Proceedings of the 11th European Particle Accelerator Conference, Genoa, Italy, 2008, paper MOPC078.

[18] D. Reschke, et al., in: Proceedings of the 13th International Workshop on RF Superconductivity, Beijing, China, 2007, paper TUP48.

[19] F. Stephan, et al., in: Proceedings of the 24th Linear Accelerator Conference, British Columbia, Canada, 2008, paper TUP035.

[20] I. Will, G. Klemz, Optics Express 16 (2008) 14923.

[21] M. Ferrario, K. Flöttmann, B. Grigoryan, T. Limberg, P. Piot, Conceptual Design of the XFEL Photoinjector, TESLA FEL Report 2001-03, DESY, Hamburg, 2001.

[22] M. Krasilnikov, et al., in: Proceedings of the 23rd Particle Accelerator Conference, Vancouver, Canada, 2009, paper MO6RFP057.

[23] Hamamutsu Femtosecond Streak Camera C6138 (FESCA-200), Hamamatsu Photonics K.K., Systems Division, 812 Joko-cho, Higashi-ku, Hamamatsu City, 431-3196, Japan〈http://www.hamamatsu.com 〉.

[24] L. Staykov, Characterization of the Transverse Phase Space at the Photoinjector Test Facility in DESY, Zeuthen Site, Ph.D. Thesis, University of Hamburg, 2008.

[25] J. Rosenzweig, G. Travish, Design Considerations for the UCLA PBPL Slit-based Phase Space Measurement Systems, PBPL Internal Note, UCLA, Department of Physics, 1994.

[26] L. Staykov, in: Proceedings of 7th European Workshop on Beam Diagnostics and Instrument for Particle Accelerators, Lyon, France, 2005, paper POT032.

[27] A. Shapovalov, L Staykov, in: Proceedings of 29th International Free Electron laser Conference, Santa Fe, New Mexico USA, 2010, paper TUPSM057.

[28] A. Brachmann, et al., in: Proceedings of the 31st International Free Electron Laser Conference, Liverpool, UK, 2009, paper WEOA03.

[29] F. Stephan, M. Krasilnikov, Invited Talk at the ICFA Workshop on The Physics and Applications of High Brightness Electron Beams 2009, Maui, Hawaii, USA, 2009.

[30] K. Flöttmann, ASTRA Particle Tracking Code $\langle$ http://www.desy.de/ $\sim$ mpyflo/ $\rangle$.

[31] K. Flöttmann, Note on the Thermal Emittance of Electrons Emitted by Cesium Telluride Photo Cathodes, Report TESLA-FEL 97-01, DESY, Hamburg, 1997.

[32] M. Khojoyan, et al., in: Proceedings of the 32nd International Free Electron Laser Conference, Malmö, Sweden, 2010, paper WEPB06.

[33] S. Schreiber, et al., in: Proceedings of the 33rd International Free Electron Laser Conference, Shanghai, China, 2011, paper TUPB04.

[34] J. Bähr, et al., in: Proceedings of the Beam Instrumentation Workshop, Santa Fe, New Mexico USA, 2010, paper TUPSM103. 